\title{
Sequential propagation and routing of activity in a cortical network
}

\author{
Juan Luis Riquelme ${ }^{1,2}$, Mike Hemberger ${ }^{1}$, Gilles Laurent ${ }^{1}$, Julijana Gjorgjieva ${ }^{1,2, *}$ \\ ${ }^{1}$ Max Planck Institute for Brain Research, Frankfurt am Main, Germany. \\ ${ }^{2}$ School of Life Sciences, Technical University of Munich, Freising, Germany. \\ *gjorgjieva@brain.mpg.de
}

\section{Abstract}

Single spikes can trigger repeatable sequences of spikes in cortical networks. The mechanisms that support reliable propagation from such small events and their functional consequences for network computations remain unclear. We investigated the conditions in which single spikes trigger reliable and temporally precise sequences in a network model constrained by experimental measurements from turtle cortex. We examined the roles of connectivity, synaptic strength, and spontaneous activity in the generation of sequences. Sparse but strong connections support sequence propagation, while dense but weak connections modulate propagation reliability. Unsupervised clustering reveals that sequences can be decomposed into sub-sequences corresponding to divergent branches of strongly connected neurons. The sparse backbone of strong connections defines few failure points where activity can be selectively gated, enabling the controlled routing of activity. These results reveal how repeatable sequences of activity can be triggered, sustained, and controlled, with significant implications for cortical computations.

\section{Introduction}

Experimental and modeling studies have proposed that cortical circuits rely on firing rates to convey information reliably in the presence of irregular activity and intrinsic sources of noise (London et al., 2010; Renart et al., 2010; Shadlen and Newsome, 1994). This suggests that individual spikes are effectively superfluous for cortical computation. On the other hand, neural circuits can produce repeatable and temporally precise patterns of action potentials (Bair and Koch, 1996; Fellous, 2004; Fellous et al., 2004; Hahnloser et al., 2002; Wehr and Laurent, 1996; Wehr and Zador, 2003), suggesting that spike timing may also play an important role (Kumar et al., 2010; Brette, 2015; Bolding and Franks, 2017). How relevant, therefore, are single spikes for cortical computation? Because spikes are the main form of neuronal communication, understanding how networks respond to single spikes is crucial to define the building blocks of cortical computation. Indeed, experiments show that a single spike can noticeably increase network firing rates in rat barrel cortex (London et al., 2010) and trigger sequences of spikes in human cortex (Molnár et al., 2008) and turtle cortex (Hemberger et al., 2019).

The influence of single neurons has been documented in rat and mouse cortex, where single-cell stimulation has meso- and macroscopic effects on network activity, brain state, and even behavior (Brecht et al., 2004; Doron et al., 2014; Houweling and Brecht, 2008; Kwan and Dan, 2012; Wolfe et al., 2010). Similarly, recent experiments in turtle cortex have shown that one to three spikes of a single neuron can trigger activity in the surrounding network (Hemberger et al., 2019). Only containing three layers, turtle dorsal (visual) cortex is architectonically similar to mammalian olfactory cortex or hippocampus and evolutionarily linked to the six-layered mammalian neocortex (Fournier et al., 2015; Tosches et al., 2018). Moreover, it lends itself to long ex vivo experiments where local connectivity is intact. The activity triggered by electrically evoked single spikes in the turtle cortex is reliable in three 
ways: responses are repeatable across trials, the responses involve the same neurons, and their activations respect the same temporal order. Repeatable sequences of spikes have been reported across various systems in vivo, such as in replay or pre-play in rat hippocampus (Buzsáki and Tingley, 2018; Diba and Buzsáki, 2007; Dragoi and Tonegawa, 2011), rat auditory and somatosensory cortex (Luczak and MacLean, 2012; Luczak et al., 2015), mouse visual and auditory cortex (Carrillo-Reid et al., 2015; Dechery and MacLean, 2017), and human middle temporal lobe (Vaz et al., 2020). Although often linked to behavioral or sensory cues, the network mechanisms that underlie such sequences are unknown. How sequences propagate, even under irregular and seemingly noisy activity, remains a puzzle, yet it may be key to understanding the computational role of sequences and cortical computations more generally (Buzsáki and Tingley, 2018).

Several candidate theoretical frameworks based on structured connectivity could explain the reliable propagation of activity during sequences. One example is synfire chains, where divergent-convergent connectivity might connect groups of neurons that fire synchronously (Abeles, 1991; Diesmann et al., 1999; Kumar et al., 2010), or polychronous chains where transmission delays require neurons to fire in precise time-locked patterns (Izhikevich, 2006). However, experimental evidence for these connectivity structures is inconclusive (Egger et al., 2020; Ikegaya et al., 2004; Long et al., 2010). Alternatively, structured connectivity leading to sequences has been proposed to arise via the training of connections (Fiete et al., 2010; Maes et al., 2020; Rajan et al., 2016). Finally, models based on the turtle cortex, in particular, have focused on network-wide propagation of waves or the statistical properties of population activity in the form of neuronal avalanches (Nenadic et al., 2003; Shew et al., 2015). Overall, these models of cortical propagation focus primarily on coordinated population activity and have not investigated how single-neuron activation can trigger reliable firing sequences.

An ex vivo preparation combining a Multi-Electrode Array (MEA) and whole-cell patchclamp allowed the exploration of sequences evoked by a controlled trigger in turtle cortex (Hemberger et al., 2019). Although these experiments established the existence and reliability of sequences, one could not observe their propagation within the excitatory neuron population due to the large electrical distance between MEA and pyramidal cell layer (Fournier et al., 2018; Shein-Idelson et al., 2017). To investigate sequence generation, we built and explored a model network incorporating experimental measurements of network connectivity and single-neuron properties of the turtle cortex. Knowing the entire model network connectivity and its resulting activity patterns enabled us to analyze the properties of modeled sequences as a function of model parameters. We found that few strong connections support sequence propagation while many weak connections provide sequence flexibility. We examined how sequences respond to sparse contextual input, modifying the paths of propagation of network activity. Our results suggest that single spikes can reliably trigger and modify sequences and thus provide reliable yet flexible routing of information within cortical networks.

\section{Results}

\section{Model of the turtle dorsal cortex with experimentally defined constraints}

Based statistically on our experimental results (Hemberger et al., 2019), we built a network model composed of 100,000 neurons (93\% excitatory and $7 \%$ inhibitory) equivalent, by our neuronal density estimates, to a $2 \times 2 \mathrm{~mm}$ slab of turtle dorsal cortex (Fig. 1A). 
bioRxiv preprint doi: https://doi org/10.1101/2021.1221.473652; this version posted December 23, 2021. The copyright holder for this preprint (which was not certified by peer review) is the author/funder, who has granted bioRxiv a license to display the preprint in perpetuity. It is made available under aCC-BY-NC-ND 4.0 International license.

Model neurons were adaptive exponential integrate-and-fire units based on experimental evidence that excitatory (pyramidal) neurons show adaptive spiking (Hemberger et al., 2019). The median of the experimental distribution of the Adaptation Index (0.3) in the turtle cortex was well captured by previously found adaptation current parameters (Brette and Gerstner, 2005). We fitted membrane capacitance and leak conductance parameters to match membrane potential recorded under current-clamp in experiments and used the median values for our model (Fig. 1B).

As suggested by axonal arbor reconstructions of turtle cortex neurons (Hemberger et al., 2019), model neurons were connected with probabilities that decayed spatially. We fitted a Gaussian profile for the spatial decay to estimates of connection probability obtained from paired whole-cell patch-clamp recordings at multiple distances (Fig. 1C). Because our estimates of connection probabilities (Fig. 1A) were derived from "paired-patch" recordings of nearby neurons, we scaled the decay profile to match population-specific probabilities in any given disc of $200 \mu \mathrm{m}$ radius.

Direct and indirect evidence from paired-patch experiments in turtle cortex indicated the presence of rare but powerful excitatory synapses, resulting in a long-tailed distribution of excitatory postsynaptic potential (EPSP) amplitudes (Hemberger et al., 2019). We thus generated excitatory synaptic conductances in our model from a lognormal distribution fitted to the experimental data (Fig. 1D). We truncated the distribution at the conductance value corresponding to the largest recorded EPSP amplitude in our paired-patch experiments (21 $\mathrm{mV})$. Based on these experimental constraints, in the model network each excitatory neuron was connected to other excitatory neurons with a majority of weak synapses and about two connections sufficiently strong to bring the postsynaptic neuron from rest to near firing threshold (Fig. 1D inset, Fig. 1E). The actual efficacy of these rare but powerful connections depended on the conductance state of their postsynaptic neurons and thus, on ongoing network activity and the state of adaptation currents. To verify that our lognormal fit was not strongly biased by our experimental dataset, we bootstrapped our estimate for the probability of a neuron having at least one strong connection under our connectivity assumptions (Fig. 1F). We found a heavily skewed distribution, with $39 \%$ of bootstrapped fits showing a probability even higher than our model.

We generated inhibitory connections from a scaled-up copy of the distribution of excitatory conductances, resulting in a skewed distribution, as observed in other systems (lascone et al., 2020; Kajiwara et al., 2020; Rubinski and Ziv, 2015). Strengths for excitatory and inhibitory connections were independent of distance. Each model connection had a random delay between 0.5 and $2 \mathrm{~ms}$ to account for the short and reliable latency between presynaptic action potential and monosynaptic EPSPs in our paired-patch experiments.

Under ex vivo conditions, recorded neurons generated spontaneous action potentials once every few seconds and had a noisy resting membrane potential (Hemberger et al., 2019) (Fig. 1G). We modeled the source of this spontaneous activity as a white noise current sampled independently but with the same statistics for every simulated neuron. In every simulation, we sampled current parameters uniformly between 50 and 110 pA mean $\left(\mu_{\text {in }}\right)$ and $0-120 \mathrm{pA}$ standard deviation $\left(\sigma_{\text {in }}\right)$. These currents produced various levels of membrane potential fluctuations and spontaneous firing, similar to those observed experimentally (Fig. 1H). Current mean had a more substantial effect than current variance on the membrane potential variance of model neurons (Fig. 1I). This resulted from selfsustained firing in the network at sufficiently depolarizing currents (note spikes in Fig. $\mathbf{1 H}$ 
bioRxiv preprint doi: https://doi org/10.1101/2021.12 21.473652; this version posted December 23,2021 . The copyright holder for this preprint (which was not certified by peer review) is the author/funder, who has granted bioRxiv a license to display the preprint in perpetuity. It is made available under aCC-BY-NC-ND 4.0 International license.

\section{bottom left).}

In summary, we built a model constrained by biological data wherever possible but random otherwise. The model displays highly heterogeneous connectivity and provides a testbed to investigate the mechanistic underpinnings of sequence generation and propagation observed in the biological networks. 
A

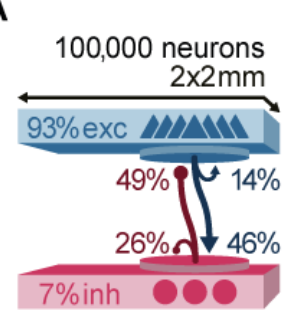

B

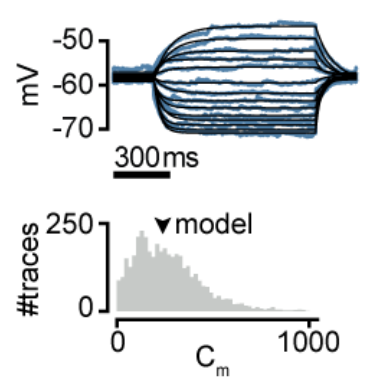

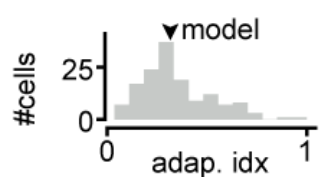

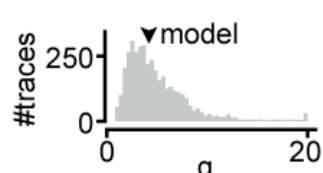

$g_{L}$
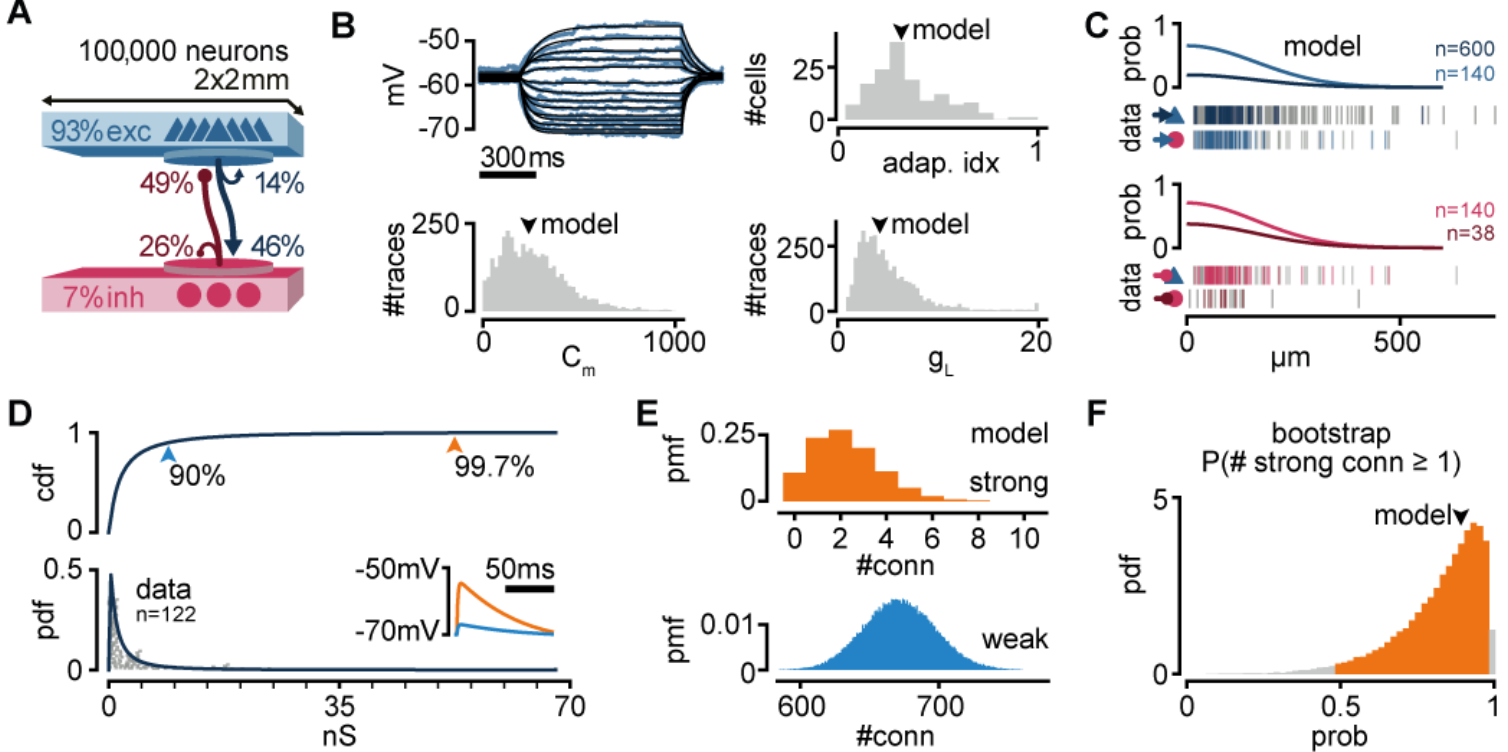

$\mathbf{F}$
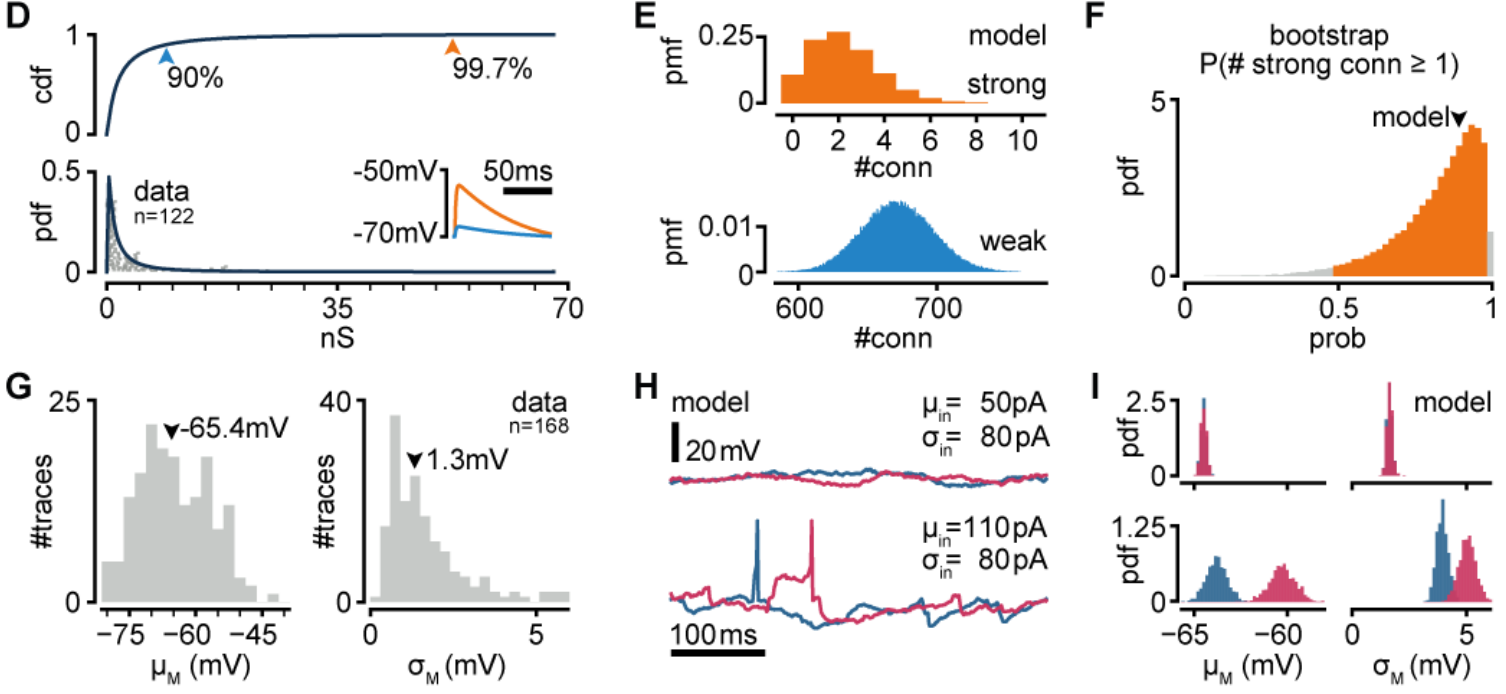

Fig. 1: A biologically inspired model of turtle dorsal cortex.

A. Schematic of the network with the size, proportion of E and I populations, and connection probabilities within a disc of $200 \mu \mathrm{m}$ radius. Blue triangles: excitatory neurons. Red circles: inhibitory neurons.

B. Top left: example of fit (black) of membrane potential responses to current injection in a recorded neuron (blue). Bottom left and right: distributions of fitted membrane capacitance $\left(C_{m}\right)$ and leak conductance $\left(g_{\mathrm{L}}\right)(n=3,886$ traces). Top right: distribution of adaptive indices for 145 recorded neurons. Arrowheads indicate parameters of model neurons (median).

C. Gaussian profiles of distance-dependent connection probabilities for different connection types (top: exc, bottom: inh). The profiles were fitted from the fraction of pairs of patched neurons that showed a connection from all tested pairs. Vertical bars below (data) indicate connected (colored) or disconnected (gray) pairs of neurons for different connection types.

D. Lognormal fit to peak excitatory synaptic conductances. Top: cumulative distribution function (cdf). Bottom: probability density function (pdf). Conductances were estimated from EPSP amplitudes experimentally obtained from recorded pairs of connected neurons (gray dots). Inset: example of modeled EPSPs for different synaptic weights (top arrowheads) in an isolated postsynaptic neuron at resting potential.

E. Probability mass function (pmf) of number of strong (top $0.3 \%$ ) or weak (bottom $90 \%$ ) excitatory-to-excitatory connections per model neuron.

F. Bootstrap estimate of the probability of an excitatory neuron having at least one strong excitatory-to-excitatory connection under our connectivity assumptions. Colored area: 95\% confidence interval. Arrowhead: fit with the original dataset in $\mathbf{D}$.

G. Experimentally measured mean and standard deviation of the membrane potential of 
bioRxiv preprint doi: https://doi org/10.1101/2021.1221 473652; this version posted December 23,2021 . The copyright holder for this preprint (which was not certified by peer review) is the author/funder, who has granted bioRxiv a license to display the preprint in perpetuity. It is made available under aCC-BY-NC-ND 4.0 International license.

168 patched neurons in ex vivo preparations. Arrowheads indicate medians.

H. Membrane potential of two model neurons under different white noise current parameters. Note the presence of action potentials and EPSPs under a high mean current $\left(\mu_{\text {in }}\right)$.

I. Distributions of membrane potential mean and standard deviation for model neurons under the white noise current parameters in $\mathbf{H}$.

\section{Reliable sequential activity in the model cortical circuit}

We next examined if our model produces the reliable sequential neuron activation from a single initial spike in a single randomly chosen pyramidal neuron (Hemberger et al., 2019). We first generated 300 random networks and randomly selected an excitatory neuron in each one (called trigger neuron). For each network, we generated 20 simulations under different levels of spontaneous activity, yielding a total of 6,000 simulations. In each simulation, we caused the trigger neuron to fire 100 action potentials at long, regular intervals (each action potential defining a trial) and measured, in all the other neurons, the resulting firing rate modulation ( $\triangle \mathrm{FR}$, Fig. 2A). To identify reliably activated model neurons, we performed a statistical test on the distribution of $\Delta \mathrm{FR}$, assuming a Poisson process of constant firing rate as the null distribution. We call "followers" those model neurons displaying statistically high $\Delta \mathrm{FR}\left(p=10^{-7}\right.$, Fig. 2B). We found followers in $94.6 \%$ of our 6,000 simulations.

When ordering the followers in the model network by activation delay from the trigger neuron spike, we observed reliable spike sequences as seen in experiments (Fig. 2C) and with similar spike rank entropy measures (see Methods, Fig. 2D). As in experiments, model sequences evolved over space, spreading away from the trigger neuron (Fig. 2E left) and did so well beyond the boundaries of our experimental measurements (defined by a 1.3x1.3mm MEA, stippled line, Fig. 2E right). Around $18 \%$ of all model followers fell outside the frame of the experimental recordings, suggesting a possible experimental underestimation of sequence size.

The number of excitatory followers far exceeded the number of inhibitory followers (Fig. $2 F)$. We tested the effect of the level of spontaneous activity in the network on the number of followers. Our model generated almost no followers when spontaneous firing rates were close to zero. The number of model followers then increased rapidly, peaking at low firing rates $(0.007 \mathrm{~Hz}$ mean firing rate for the top $1 \%$ simulations by follower count), and slowly declined as spontaneous firing rates increased. We classified our simulations into low and high activity using spontaneous mean firing rate ranges observed in experiments: [0, 0.05] $\mathrm{Hz}$ ex vivo (Hemberger et al., 2019), 41\% of simulations; [0.02, 0.09] Hz in vivo (Fournier et al., 2018$), 33 \%$ of simulations $\left(\left[25^{\text {th }}, 75^{\text {th }}\right]\right.$ percentiles).

We found sequences in both groups but with notable differences in the behavior of excitatory and inhibitory model followers. The probability of a low-activity simulation displaying at least 1 or 10 inhibitory followers (74\% and $19 \%$ respectively) was in excellent agreement with experimental data ( $77 \%$ and $20 \%$ respectively, Fig. 2 G). Interestingly, the probability of producing inhibitory followers dropped in high-activity simulations (4\% prob. of $\geq 10$ inhibitory followers). Excitatory neurons showed the opposite trend: the probability of having at least 10 excitatory followers was higher in high-activity (69\%) than in low-activity simulations (50\%). 
The sequences of activations of excitatory followers often lasted more than $150 \mathrm{~ms}$ and reached the spatial boundaries of our model circuit $(2 \times 2 \mathrm{~mm}, 100 \mathrm{k}$ neurons) in both low- and high-activity simulations (Fig. 2H left). Inhibitory followers in the model network, however, often fired early in the sequence and were spatially closer to the trigger neuron than excitatory ones (Fig. 2H middle and right). These differences were more pronounced in high- than low-activity simulations (Fig. 2H, gray and colored). Importantly, inhibitory followers displayed near-random levels of temporal jitter in high-activity regimes (Fig. $\mathbf{2 H}$, foll. jitter, standard deviation of spike delays). Consequently, we expect followers to be detectable in experiments in vivo but mainly within the pyramidal layer.

In summary, our model produced repeatable firing sequences across groups of neurons in response to single spikes in randomly chosen excitatory neurons with properties very similar to those observed in ex vivo experiments that constrained the model network. Our simulations predict that sequences can occur under in vivo levels of spontaneous activity. In these conditions of higher firing rates, the activation sequences are mainly composed of excitatory followers, whereas inhibitory followers produce less reliable and temporally jittered responses. 

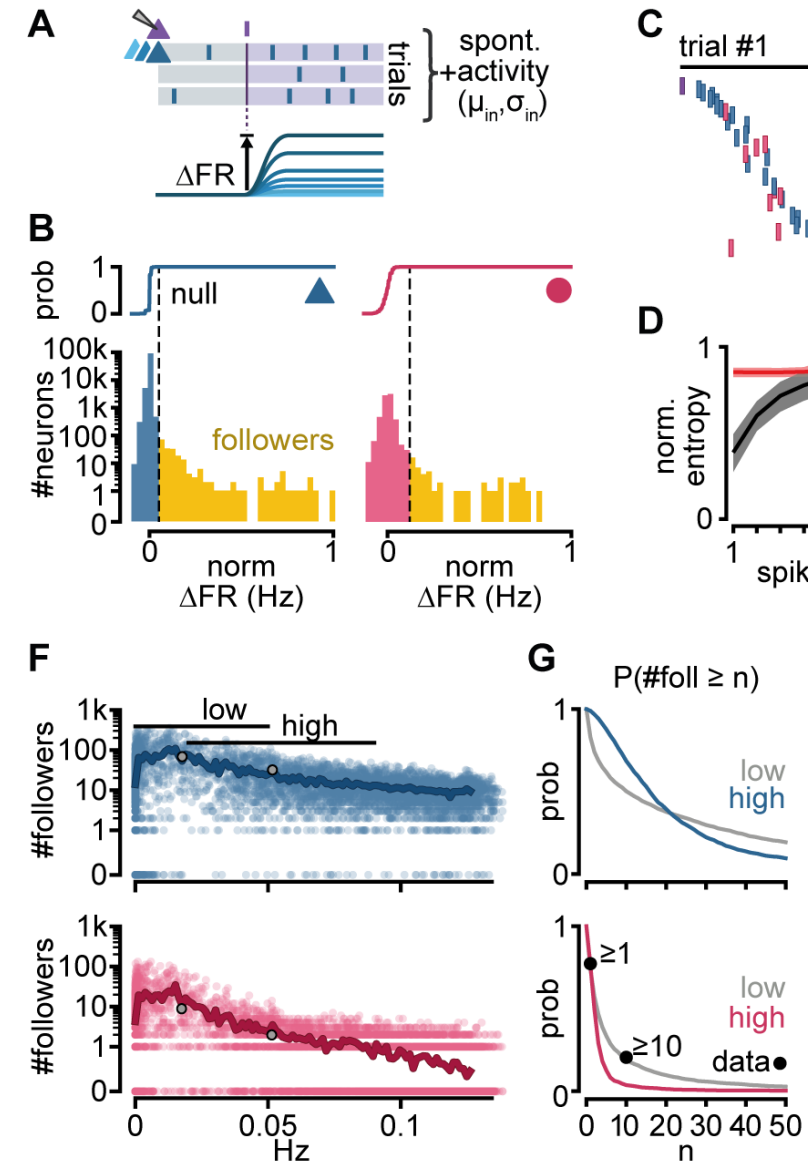
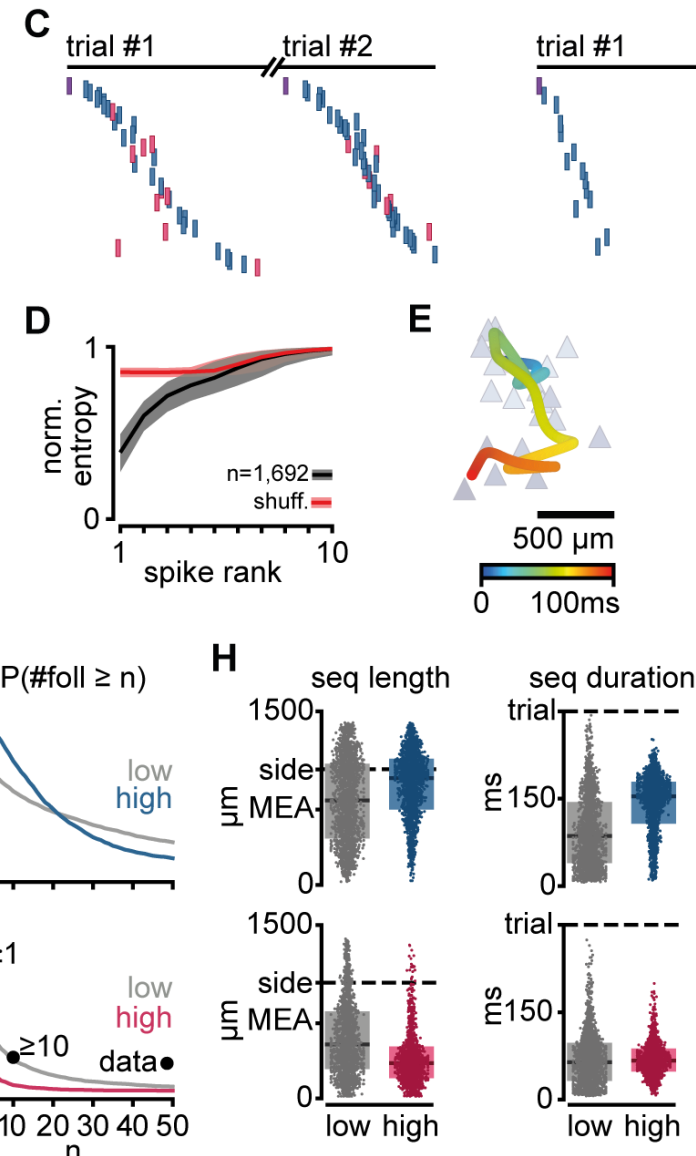
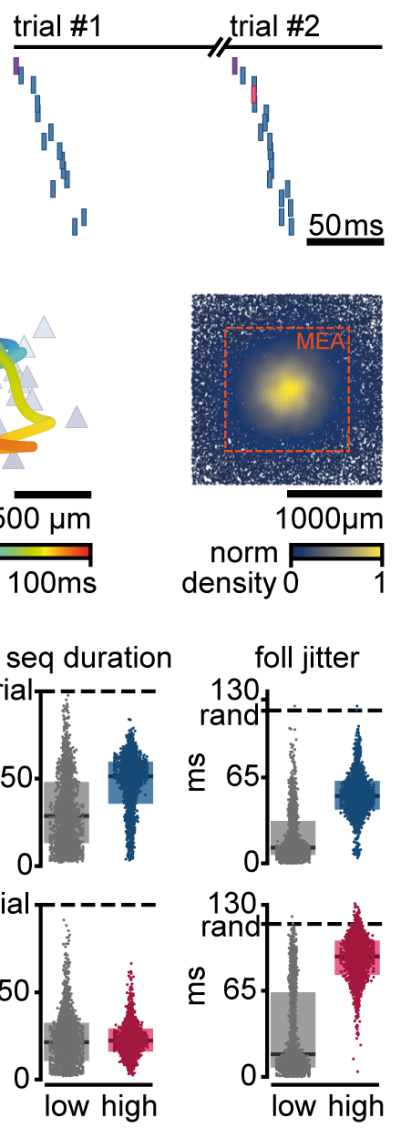

Fig. 2: Repeatable sequential activation of follower neurons in the model network.

A. Schematic of the stimulation protocol in the model. One neuron (top) was driven to produce one action potential, and all other neurons in the network were tested for resulting changes in their firing rate.

B. Distribution of single-cell firing rate modulation $(\Delta \mathrm{FR})$ for one example simulation. Top: cumulative mass function of the null distribution. Dashed: threshold for follower classification $\left(p=10^{-7}\right)$. Bottom: $\triangle F R$ for followers (yellow) and other neurons in the network (blue: exc, red: inh). $\Delta \mathrm{FR}$ is normalized to the modulation of a perfect follower (exactly one spike per trial).

C. Left: Sequence of spikes from followers in two consecutive trials in an example simulation. Neurons are sorted by median spike-time across all trials. Same sorting in both trials. Spikes from non-followers are not shown. Right: same for a different simulation with a higher mean firing rate (left: $0.017 \mathrm{~Hz}$, right: $0.051 \mathrm{~Hz}$ ).

D. Normalized spike rank entropy of sequences with at least 10 followers (black: mean; gray: std), compared to shuffled sequences (red).

E. Left: Example center-of-mass of follower activations corresponding to the first sequence in C. Right: spatial location of excitatory followers pooled from all simulations, colored by local density $(n=117,427)$. All simulations are aligned so that the trigger neuron is in the center. Stippled square: size of the MEA used in experiments.

F. Number of followers detected for each simulation as a function of the mean level of activity in the network. Blue: exc; red: inh; thick line: moving average; Gray dots:

sequences in $\mathbf{C}$. 
bioRxiv preprint doi: https://doi org/10.1101/2021.1221 473652; this version posted December 23, 2021. The copyright holder for this preprint (which was not certified by peer review) is the author/funder, who has granted bioRxiv a license to display the preprint in perpetuity. It is made available under aCC-BY-NC-ND 4.0 International license.

G. Probability of generating a minimum number of followers for excitatory and inhibitory populations in high- and low-activity simulations. Dots: experimental ex vivo estimates. H. Statistics of excitatory- or inhibitory-follower activations by activity level. Left: Distance from trigger neuron to farthest detected follower in each simulation (side: half-width of the model network; MEA: half-width of MEA used in experiments). Middle: Delay to median spike-time of the last activated follower in each simulation (trial: maximum detectable duration under protocol in A). Right: standard deviation of follower spike times, averaged over all followers in each simulation (rand: expected standard deviation of randomly distributed spike times). Boxes: median and $\left[25^{\text {th }}, 75^{\text {th }}\right]$ percentiles.

\section{Distinct roles of strong and weak connections in the propagation of activity in the network}

To better understand the mechanisms behind follower activation, we examined how action potentials propagate through our model networks. In a random subset of our 6,000 simulations $(n=900)$, we searched for instances when an excitatory neuron spike successfully traversed a connection, i. e. whenever both pre- and postsynaptic neurons fired within a short interval (100 ms). We call these "spike transfers". We thus combined action potentials with recurrent connectivity to produce a directed acyclic graph of spike transfers for each simulation (Fig. 3A). Most excitatory-to-excitatory spike transfers in low-activity simulations occurred within 6-8 ms (Fig. 3B), matching delays measured in turtle cortex (Hemberger et al., 2019). Interestingly, excitatory-to-inhibitory spike transfers were consistently shorter than their excitatory-to-excitatory counterparts, even at higher firing rates (Fig. 3B inset), possibly reflecting the more depolarized state of inhibitory neurons (Fig. 1H right).

To understand the connectivity structure underlying these spike transfers, we examined connectivity motifs in the graph (Fig. $\mathbf{3 C}$ ). The possible number of motifs increases with the number of neurons involved. Due to this combinatorial nature, we extracted only low-order motifs with depths of up to two spike transfers and involving $\leq 4$ spikes. In low-activity simulations, very few motifs led to the activation of excitatory neurons. The number of oneto-one excitatory spike transfers (Fig. $3 \mathrm{C}$ top left, single) was higher than that resulting from convergence (Fig. $3 \mathrm{C}$ top left, conv.). By contrast, spikes from inhibitory neurons resulted from more spike transfers, with a prevalence of convergence motifs (Fig. 3C top right, conv.). Although spike transfers by convergence became more common in high-activity simulations for both excitatory and inhibitory populations (Fig. $3 \mathrm{C}$ bottom), the increase was greater for motifs leading to inhibitory spikes. Indeed, inhibitory spikes in high-activity simulations rarely involved single spike transfers (Fig. 3C bottom right, single).

Finally, we extracted the synaptic strengths involved in these spike transfers (Fig. 3D). The synaptic strength distribution underlying spike transfers was long-tailed, matching the static connectivity of our networks (Fig. 1D). Interestingly, the distribution of excitatory-toexcitatory spike transfers was bimodal, with an over-representation of strong connections (Fig. 3D top inset). By contrast, excitatory-to-inhibitory spike transfers relied primarily on weaker connections (Fig. 3D bottom). The difference between the two populations was again greater at higher levels of activity.

This suggests that excitatory and inhibitory neurons participate in different types of motifs and with different synaptic strengths to propagate sequences. First, inhibitory neurons were usually activated by the convergent activation of multiple weak connections. The low fraction 
bioRxiv preprint doi: https://doi.org/10.1101/2021.12 21.473652 this version posted December 23, 2021. The copyright holder for this preprint (which was not certified by peer review) is the author/funder, who has granted bioRxiv a license to display the preprint in perpetuity. It is made available under aCC-BY-NC-ND 4.0 International license.

of inhibitory neurons and the high E-to-I connection probability (Fig. 1A) made inhibitory neurons strongly susceptible to spontaneous network activity. Consequently, inhibitory neurons were less selective to their excitatory drivers, limiting their capacity to become followers. Second, excitatory neurons were less susceptible to ongoing network activity than their inhibitory counterparts. Single strong excitatory inputs were the dominant drive behind excitatory spikes and thus, the likely conduit of sequence propagation.

To confirm the role of strong connections in sequence propagation, we built variations of our model in which we removed connections according to their strengths (Fig. 3E top). We defined weak and strong connections as, respectively, the bottom $90 \%$ or top $0.3 \%$ of all connections, representing the two modes of the distribution of synaptic strengths of excitatory-to-excitatory spike transfers (Fig. 3D top). We then re-ran a random subset of all simulations using the modified models $(n=2,000)$. Networks with only weak connections required a much stronger drive than their intact counterparts to produce any activity (Supplementary Fig. 1A) and produced fewer excitatory followers ( $0 \%$ prob. of $\geq 10$ followers, Supplementary Fig. 1C), in contrast with those with only strong connections (75\%) (Fig. 3E bottom). Strong connections were thus necessary and sufficient to produce repeatable sequences in our model.

Interestingly, the model containing only strong connections often produced more followers, suggesting that weak connections affect the reliability and thus the detection of potential followers. Indeed, eliminating weak connections reduced the slope of our network input/output (I/O) curve (Fig. 3F). Consequently, networks with only strong connections remained at low firing rates for a broader range of inputs, in which more followers could be identified (Fig. 2G). Eliminating weak connections also caused a shift of the I/O curve to the right, increasing the range of inputs over which output activity was close to zero. This shift defines a narrow range of inputs where the presence of weak connections, instead of reducing the number of followers, increases it (Fig. 3G).

In summary, rare but strong and common but weak connections play different roles: the former promote reliable responses to single spikes, while the latter amplify spontaneous network activity and drive recurrent inhibition, effectively modulating the reliability of those responses. 
A

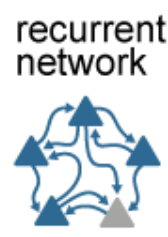

spike transfers

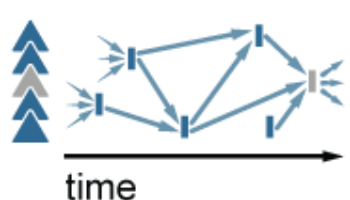

C
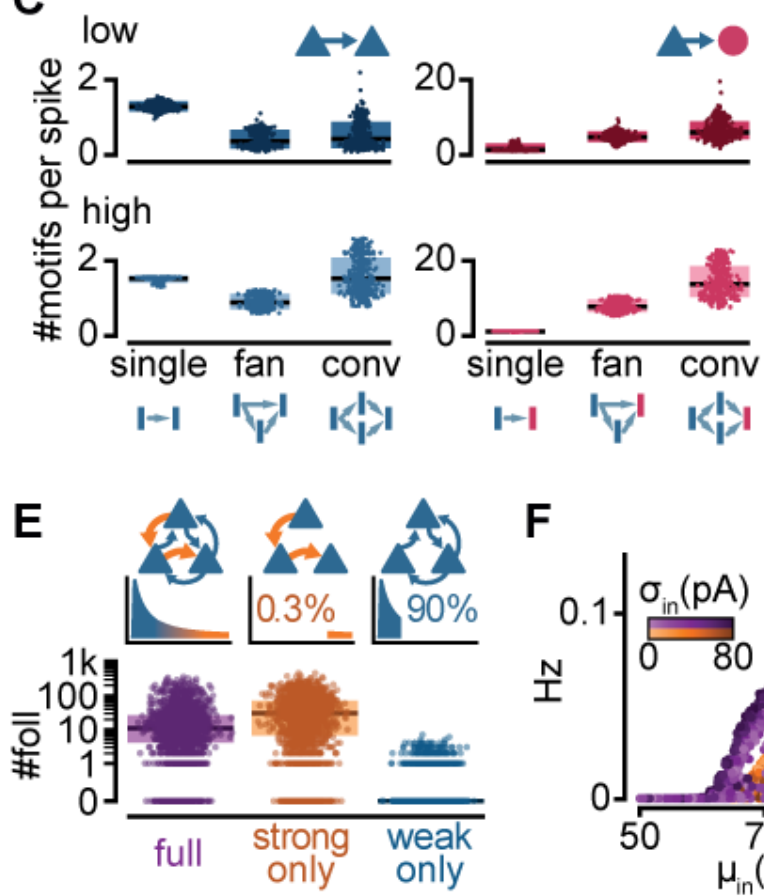

$\mathbf{F}$
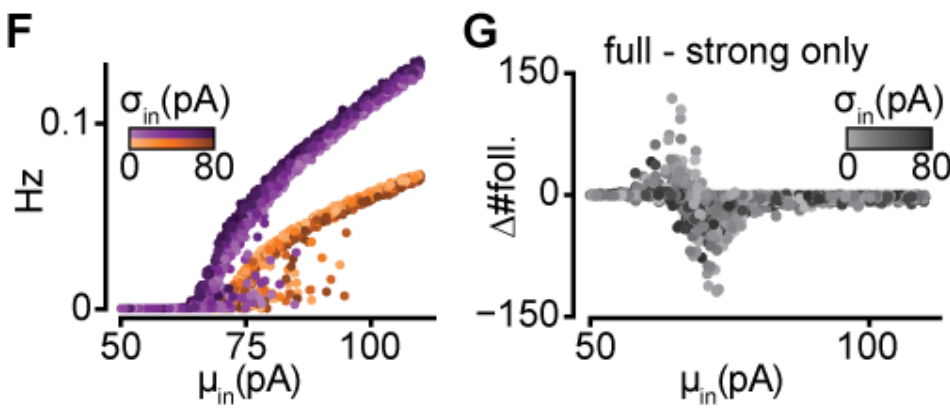

Fig. 3: Connectivity underlying activity propagation in the network.

A. Schematic. The recurrent network structure (left) and spiking activity are combined to produce a directed graph of spike transfers (right).

B. Distribution of delays of excitatory-to-inhibitory (red) and excitatory-to-excitatory (blue) spike transfers. Average of all low-activity simulations $([0,0.05] \mathrm{Hz})$. Arrowheads: mode of each distribution. Inset: Modes for average distributions grouped in equal bins of mean firing rate.

C. Number of detected motifs leading to an excitatory (blue) or inhibitory (red) neuron spike. Each dot represents the mean for all spikes in each simulation. Simulations are grouped by low (top) or high (bottom) activity. Boxes: median and $\left[25^{\text {th }}, 75^{\text {th }}\right]$ percentiles. Conv: convergence.

D. Histograms of strengths of synapses leading to excitatory (blue) or inhibitory (red) neuron spikes. Insets: zoomed-in tails. Each line averages 25-100 simulations grouped in equal bins of mean firing rate (color bar).

E. Top: schematic of alternative network models and their truncated distribution of synaptic strengths. Bottom: Number of detected excitatory followers per simulation ( $n=2,000$ each). Boxes: median and $\left[25^{\text {th }}, 75^{\text {th }}\right]$ percentiles.

F. Input-output curves for the full (purple) and strong-only (orange) models.

G. Difference between the number of followers detected in full and strong-only models under the same input. 
bioRxiv preprint doi: https:/doi org/10.1101/2021.1221.473652; this version posted December 23,2021 . The copyright holder for this preprint (which was not certified by peer review) is the author/funder, who has granted bioRxiv a license to display the preprint in perpetuity. It is made available under aCC-BY-NC-ND 4.0 International license.

\section{Sequences are composed of sub-sequences that correspond to sub-networks of strong connections}

To better understand the regulation of sequential neuronal activations, we examined when and how sequences fail to propagate. To this end, we chose a simulation with an intermediate firing rate $(0.034 \mathrm{~Hz})$, compatible with both ex vivo and in vivo conditions, and follower count (25) closest to the average for that firing rate. Over repeated trials with the same trigger (Fig. 4A columns), we observed that followers (Fig. 4A rows) differed from one another in their reliability. As a result, the exact spiking composition of a sequence could vary from one trial to the next, consistent with our definition of followers as neurons activated in a significant fraction of, but not necessarily all, trials. Because follower activations depended mainly on rare strong connections (Fig. 3E), we reasoned that the activation or failure of any follower could affect the unfolding of a sequence. Consequently, we performed k-modes clustering of followers based on their activation over trials (Fig. 4B left). Our analysis revealed clusters composed of followers that tended to activate together in the same trials (Fig. 4C left). When we mapped these follower clusters onto the graph of connections, we found that the connections within each cluster were strong, and belonged to the tail of the distribution of connection strengths (Fig. 4B right, Fig. 4C right). We thus call each one of these follower clusters "sub-networks", and their corresponding sections of the spiking sequence "sub-sequences". We observed similar decompositions into subsequences across simulations with different trigger neurons and number of followers (Fig. 4D). Across all simulations, strong connections (in the top $0.3 \%$ of the distribution) were more frequent within than between sub-networks, providing a mechanistic substrate for subnetwork segregation (Fig. 4E). Furthermore, when strong connections existed between subnetworks, they did not always guarantee the reliable propagation of activity between them (Fig. 4F).

To further examine the potential interdependence of sub-network activation, we selected the two largest sub-networks of every simulation ( $a$ and $b$ ) and classified each trial as one of four possible outcomes: full failure, $a$ and $b$ together, $a$ alone, or $b$ alone (Fig. 4G top). We defined a sub-network as activated if at least $40 \%$ of its followers fired at least once in that trial. We then extracted the mean entropy of these outcomes as a function of network mean firing rate across all simulations with at least two sub-networks (Fig. 4G bottom). The mean entropy was about 1 bit at low firing rates, indicating two equally likely outcomes (full failure or partial propagation). As spontaneous activity increased, entropy rose towards its maximum (2 bits), where all four outcomes became equally likely. This trend towards maximal entropy is consistent with recurrent connectivity and spontaneous activity in the model network being random, which, on average, avoids biases of activation of one subnetwork over the other.

In summary, sequence decomposition based on coactivity revealed sub-networks of strongly connected followers, linked to each other by weak or unreliable connections. These sub-networks could act as independent paths for the propagation of activity. 
A
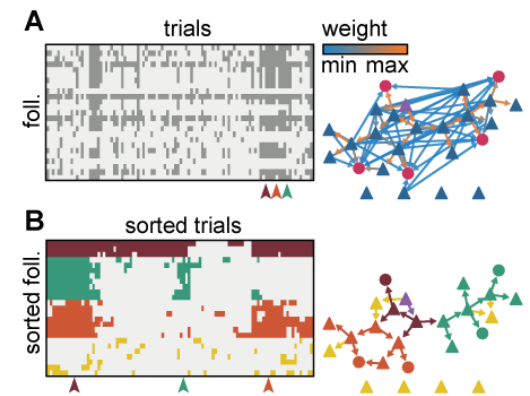

C

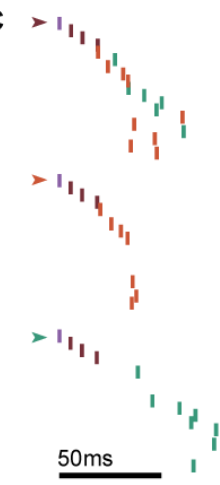

D
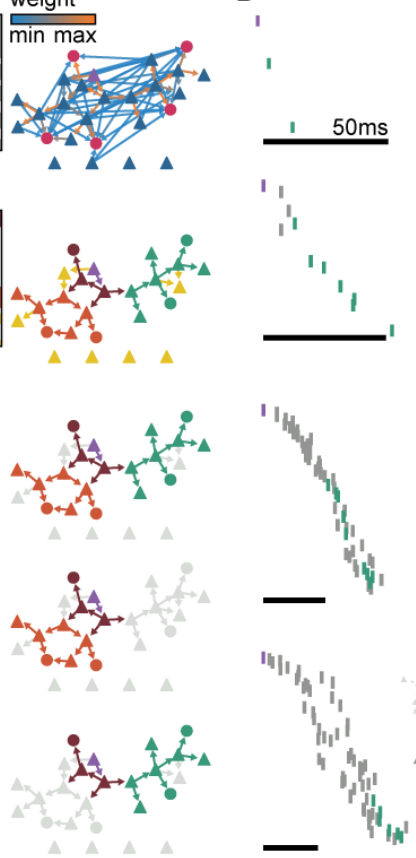
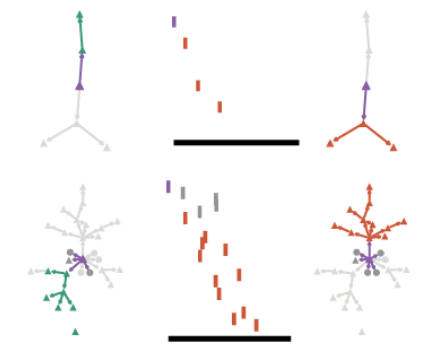

E

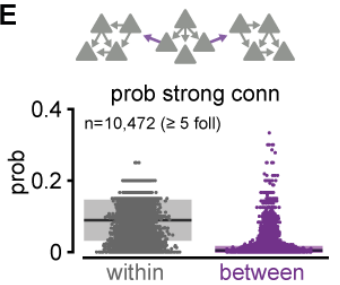

$\mathbf{F}$

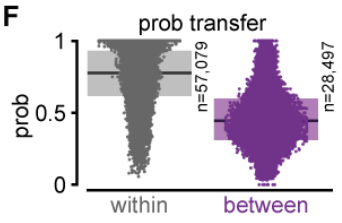

G

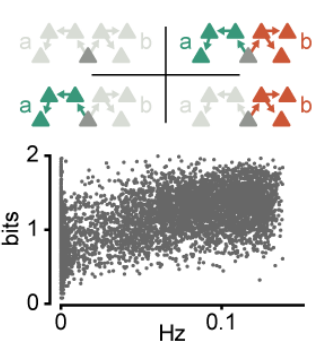

Fig. 4: Clusters of sequential spikes reflect sub-networks of strongly connected followers.

A. Left: Activation (dark matrix entries) of followers (rows) over repeated trials (columns) of an example simulation. Colored arrowheads indicate trials in $\mathbf{C}$. Not all followers activate in all trials. Right: Graph of excitatory follower-to-follower connections colored by strength. Trigger neuron in purple. Neurons identified as followers but not directly connected are aligned below.

B. Left: Matrix in A after sorting followers and trials according to k-modes clustering. Right: same graph as in A, with only top $5 \%$ connections. Colors according to follower clustering. Note that members of orange, green, and brown clusters are connected to one another.

C. The three trials indicated in A and B. Left: firing sequence with spikes colored according to their follower cluster. Right: same graph as in $\mathbf{B}$, with inactive followers in light gray. D. Two trials (columns) of four different simulations to illustrate selective sub-network activation. Top to bottom: different trigger neurons with 5, 28, 68, and 145 followers. Only top $5 \%$ connections are shown. Followers were clustered as in B. Green and orange: example active sub-networks. Dark gray: other active sub-networks. Light gray: inactive followers.

E. Relationship between connectivity and follower clusters. Top: schematic illustrating connections between (purple) and within (gray) clusters. Bottom: Probability of strong (top $0.3 \%$ ) excitatory-to-excitatory connection within or between clusters, for 10,472 clusters of at least 5 followers pooled across all 6,000 simulations.

F. Probability of postsynaptic activation conditioned on presynaptic activation in the same trial, for excitatory-to-excitatory connections, pooled across all 6,000 simulations. Boxes: median and $\left[25^{\text {th }}, 75^{\text {th }}\right]$ percentiles.

G. Relationship between mean entropy and baseline firing rate. Top: schematic of four possible propagation scenarios in a simplified scheme where only two sub-networks are considered per simulation. Bottom: Mean entropy over trials for each simulation with at least 2 sub-networks $(n=5,371)$ as a function of network mean firing rate. 
bioRxiv preprint doi: https://doi org/10.1101/2021.1221.473652; this version posted December 23,2021 . The copyright holder for this preprint (which was not certified by peer review) is the author/funder, who has granted bioRxiv a license to display the preprint in perpetuity. It is made available under aCC-BY-NC-ND 4.0 International license.

\section{External input can halt or facilitate the propagation of activity}

We hypothesized that the connections between connected sub-networks could be critically important for sequence propagation. Indeed, the excitability and state of an entry point to a sub-network should affect sub-network activation, hence, we refer to these entry point neurons as "gates". We identified the gates for every sub-network of every simulation based on activity, as the neurons within each sub-network with the shortest median spike delay from trigger.

To evaluate how the excitability of gate neurons might affect propagation in the rest of the sub-network, we varied their level of excitatory or inhibitory conductances. We then ran again a random selection of our original simulations $(n=2,000)$ but this time, a randomly selected gate received an additional action potential from an external source (Fig. 5A). The conductance of this external input was the maximum value from either the excitatory or inhibitory synaptic conductance distribution (Fig. 1D). The level of spontaneous activity in the rest of the network was unchanged. We defined the level of activation of each subnetwork as the fraction of trials (out of 100 ) in which the sub-network was active, and then computed the change of activation, relative to control, under the altered conductance state of the gate $\left(\Delta \mathrm{a} / \mathrm{a}_{0}, \%\right)$ (Fig. 5B). We found that external inputs to gate neurons were highly effective in controlling propagation: a single external inhibitory-input spike halved subnetwork activation probability in $74 \%$ of the simulations and entirely halted its activation in $26 \%$ of the simulations. By contrast, a single external excitatory-input spike doubled baseline sub-network activation probability in $55 \%$ of the simulations (Fig. 5B, Supplementary Fig. 2A).

To examine the effect of timing and strength of external input on sub-network activation, we focused on the network in Fig. 4A. We varied the sign (depolarizing vs. hyperpolarizing) and amplitude of the synaptic conductance of the external input to a gate neuron, as well as its timing relative to trigger activation. We explored only conductances corresponding to single connections. We observed three different effects on the activation of the respective sub-network $\left(\Delta \mathrm{a} / \mathrm{a}_{0}, \mathbf{F i g} . \mathbf{5 C}\right.$ solid outlines). First, an inhibitory input of any strength in a temporal window of $\sim 100$ ms could acutely reduce sub-network activation, often halting propagation (bottom outline). Second, an excitatory input of intermediate strength could facilitate propagation (top right outline). The temporal window of facilitation was centered on the expected spike time of the gate neuron. Lastly, an excitatory input of large amplitude could impair propagation if it occurred much earlier than the activation of the trigger neuron (top left outline). We attribute this effect to the adaptive properties of our model neurons (Fig. 1B): the external input, by triggering the gate and some of the neurons in its subnetwork, activated adaptive currents and thus raised their threshold for subsequent activation by the trigger neuron. We found equivalent effects on networks with number of followers and baseline firing rates spanning orders of magnitude (Supplementary Fig. 2BC). From the average over all tested sub-networks, we note that halting was most effective in a narrower time window (relative to trigger activation) than facilitation $(70 \mathrm{~ms}$ and $100 \mathrm{~ms}$, respectively) (Fig. 5D left). When considered relative to the expected spike time of the gate, the temporal windows became wider (Fig. 5D right). Importantly, while the halting window had a sharp temporal boundary determined by the expected spike time of the gate in the sequence, the facilitation window increased with increasing input amplitude.

Finally, we examined how modulating the gate to one sub-network affected the activation of another (Fig. 5E). Interestingly, this relationship between sub-networks was not 
symmetrical: in this network example, while orange sub-network activation was primarily independent of the state of the green sub-network (Fig. 5E left), gating of the orange subnetwork unexpectedly facilitated green sub-network activation (Fig. 5E right, bottom outline), suggesting that the former typically exerts a net inhibitory influence on the latter. Indeed, strong excitatory inputs that might activate the orange sub-network early also reduced green sub-network activation (Fig. 5E right, top outline). We observed complex interactions between pairs of sub-networks for other networks (Supplementary Fig. 2B right) but no strong trend on average (Supplementary Fig. 2C), suggesting that subnetwork interactions were idiosyncratic, i.e., dependent on the particular realization of the random connectivity in the network.

In short, temporally constrained external control of the state of gate neurons can halt or facilitate the activation of particular sub-networks or sets of strongly connected neurons. Recurrent connectivity can also lead to complex and non-reciprocal interactions between sub-networks. 
A
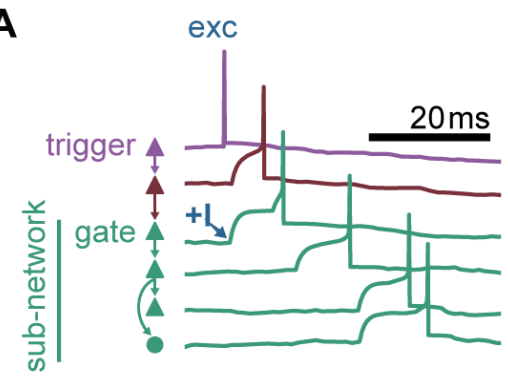

inh

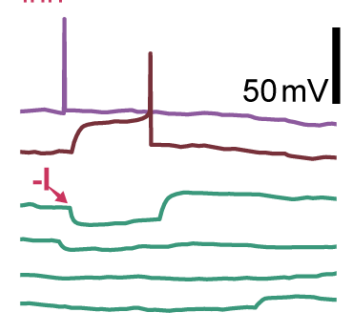

C

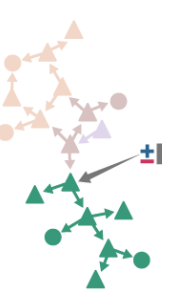

D
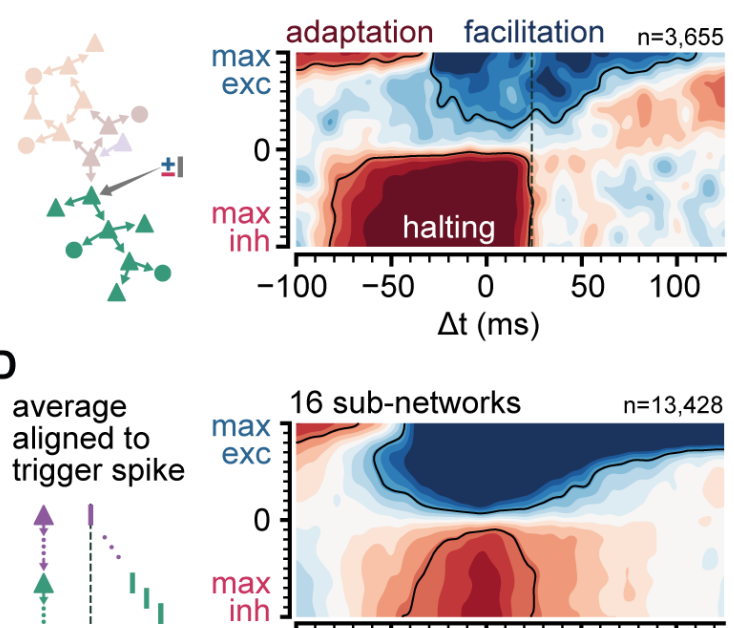

$\Delta \mathrm{t}(\mathrm{ms})$
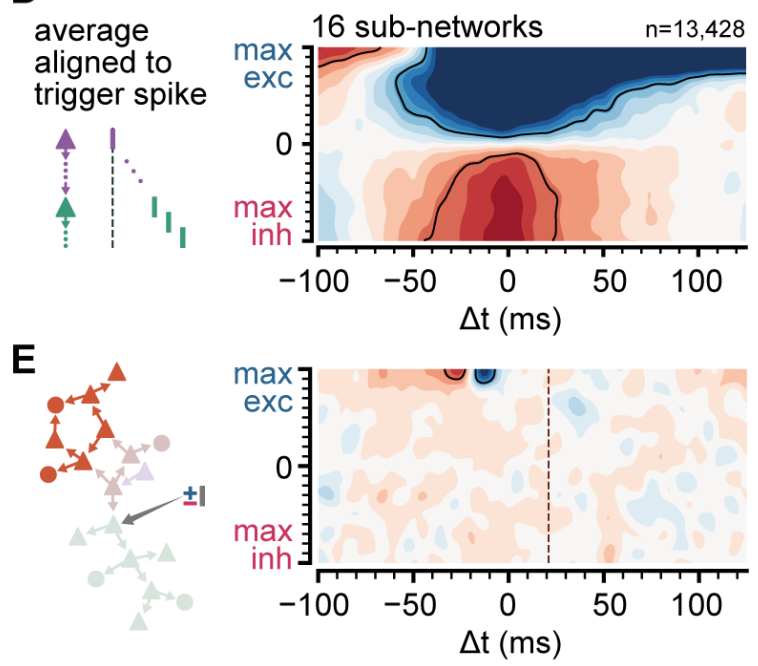

B
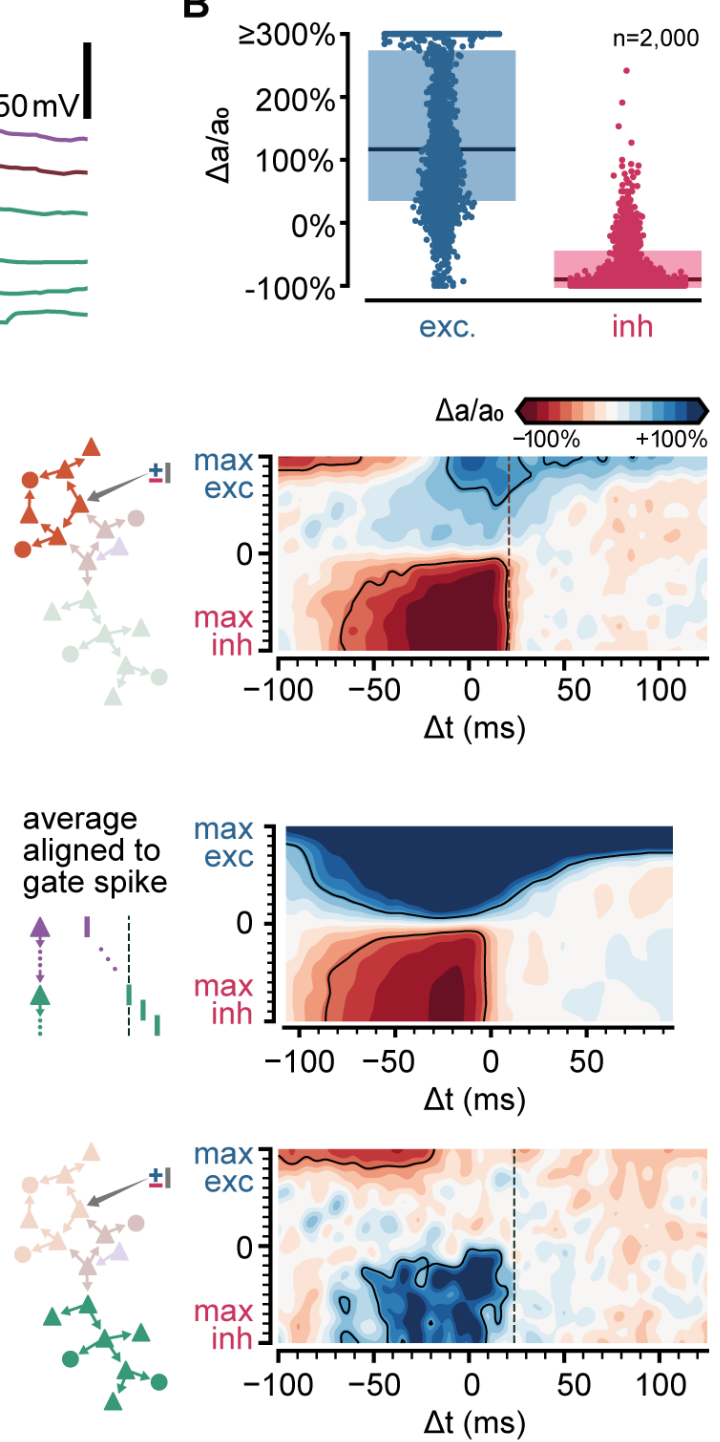

Fig. 5: Sub-sequence activations depend on the state of gate neurons.

A. Voltage traces for 6 neurons in a sequence (network and colors as in Fig. 4A) in two trials of simulations where the first neuron in the green sub-network receives a single excitatory (left, $+\mathrm{I}$ ) or inhibitory (right, $-\mathrm{I}$ ) input from an external source (arrows).

B. Fold change of sub-network activation above baseline $\left(\Delta \mathrm{a}_{\mathrm{a}} \mathrm{a}_{0}, \%\right)$ for sub-networks randomly selected across all 6,000 original simulations. The model gate neuron of each sub-network received an additional single input from an external source at the beginning of each trial.

C. Schematic of protocol and map of the change in activation $\left(\Delta a_{a} / a_{0}, \%\right)$ for each of the two sub-networks (left: green, right: orange, same network as in Fig. 4A) when manipulating the gate to that sub-network. Arrow in schematic indicates stimulated gate. Solid outlines indicate combinations of strength and timing leading to halting (bottom, $-50 \%$ $\Delta \mathrm{a} / \mathrm{a}_{0}$ ), facilitation (top right, $+50 \% \Delta \mathrm{a} / \mathrm{a}_{0}$ ) and adaptation (top left, $-50 \% \Delta \mathrm{a} / \mathrm{a}_{0}$ ). Dashed lines indicate the median spike time of the respective gate.

D. Average map of the change in activation $\left(\Delta \mathrm{a} / \mathrm{a}_{0}, \%\right.$, same as $\left.\mathbf{C}\right)$ computed from 16 subnetworks of 8 different networks spanning 5-462 followers and $0.01-0.1 \mathrm{~Hz}$ baseline firing rate (networks in $\mathbf{C}$ and Supplementary Fig. 2B). Left: maps were computed relative to 
bioRxiv preprint doi: https://doi org/10.1101/2021.1221.473652; this version posted December 23, 2021. The copyright holder for this preprint (which was not certified by peer review) is the author/funder, who has granted bioRxiv a license to display the preprint in perpetuity. It is made available under aCC-BY-NC-ND 4.0 International license.

trigger spike before averaging. Right: maps were computed relative to each gate median spike time before averaging. Solid outlines indicate $\pm 50 \% \Delta \mathrm{a} / \mathrm{a}_{0}$. Color bar as in C.

E. Left: same as $\mathbf{C}$ but concerning the orange sub-network when manipulating the green gate. Right: same for the green sub-network when manipulating the orange gate.

\section{Combinatorial activation of follower sub-networks via intra-cortical interactions}

To examine how sequences interact, we studied a new set of simulations where multiple trigger neurons were activated simultaneously. We first simulated 100 trials by inducing a single spike from each of 2,000 randomly selected trigger neurons in a representative network (as in Fig. 4A, though see Supplementary Fig. 3 for multiple network instantiations). Randomly pairing these trigger neurons, we estimated a very low probability that two excitatory neurons share followers (Fig. 6A, Fig. 6B). Two triggers shared at least 1 follower in only $3.12 \%$ of all tested pairs $(n=5,000)$, and this probability dropped to $0 \%$ for at least 11 followers.

Next, we generated a new simulation for each randomly chosen pair of trigger neurons, in which both were activated simultaneously (100 trials). Comparing the followers resulting from these double-trigger activations to the followers from single-trigger activations revealed three sets of followers (Fig. $\mathbf{6 C}$ ): ones responsive to the activation of only one of the triggers (a), followers responsive to the activation of only the other trigger (b), and followers responsive to their coactivation (a\&b).

The number of followers obtained under a\&b coactivation was smaller than the sum of the number of followers of $a$ and $b$ alone (Fig. 6D, Supplementary Fig. 3A). Since $a$ and $b$ rarely shared followers, the resulting sublinear activation suggested frequent lateral inhibition, as we had observed between sub-networks (Fig. 5E). Indeed, followers to a single trigger could be lost when that trigger was coactivated with another (a only and b only, Fig.

6E, Supplementary Fig. 3B). Other followers, however, could emerge when two trigger neurons were coactivated but were absent in response to the activation of either trigger alone (ab only). We call these "combination-specific followers" because their activation depends on whether multiple trigger neurons are silent or active (a only, b only, and ab only). Combination-specific followers represented approximately half of all followers in the coactivation protocol (Supplementary Fig. 3C). Finally, some followers fired independently of whether the second trigger neuron was silent or active (a\&ab, and b\&ab). We call these "core followers". The excitatory and inhibitory compositions of core and combination-specific followers did not differ (Supplementary Fig. 2D). Core followers typically fired early in a sequence, whereas combination-specific followers became activated later (Fig. 6F). This is consistent with our experimental and modeling findings that follower identity is more reliable early than late in a sequence (Hemberger et al., 2019) (Fig. 2D). By contrast, the late activation of combination-specific followers suggests that modulation of sequences via intracortical interactions operates best on followers synaptically distant from the trigger neuron.

These findings prompted us to examine the effect of circuit interactions on sub-network activation in a known sequence. We used the trigger in the network in Fig. 4A as our control and coactivated it with each of 2,000 randomly selected excitatory neurons in the network, which we call "contextual neurons". By contrast with random spontaneous activity, these additional activations formed a context of activity that remained constant from trial to trial. We then evaluated the effect of coactivation on the sub-networks of our control sequence (Fig. 6G-H). Most contextual neurons excited or inhibited both sub-networks equally 
bioRxiv preprint doi: https://doi org/10.1101/2021.12 21.473652 this version posted December 23, 2021. The copyright holder for this preprint (which was not certified by peer review) is the author/funder, who has granted bioRxiv a license to display the preprint in perpetuity. It is made available under aCC-BY-NC-ND 4.0 International license.

(diagonal trend, Fig. 6H). Consistent with the tendency towards lateral inhibition (Fig. 6D), $87.8 \%$ of the tested contextual neurons caused some inhibition of both sub-networks (bottom left quadrant, Fig. 6H). Lateral excitation was also present but rarer $(6.7 \%$, top right quadrant, Fig. $6 \mathbf{H}$ ). The symmetry along the diagonal was likely a consequence of our random connectivity and reflected our earlier observation that specific pairs of sub-networks can strongly influence each other, with the average effect being balanced (Supplementary Fig. 2B right, Supplementary Fig. 2C). Some contextual neurons prevented activation of one of the sub-networks while facilitating the other (top left and bottom right quadrants, Fig. $6 \mathrm{H}, 2.7 \%$, and $2.9 \%$, respectively, Supplementary Fig. 3E). The small percentage ( $6 \%$ ) of contextual neurons that could determine which sub-network became active suggests a highly specific form of routing. This result is consistent with our previous finding that excitatory followers are generally tolerant of ongoing network activity (Fig. 3C, Fig. 3D). Given the size of our model network ( $2 \times 2 \mathrm{~mm}, 100 \mathrm{k}$ neurons, Fig. 1A), we estimate that in a given trial, the path of activation through the network can be influenced by the activation of $\sim 5,000$ contextual neurons.

In short, our results indicate that overlap or criss-crossing of sequences initiated by two different trigger neurons is rare, but that interactions between the sequences are common. Sequence interactions can define followers that are highly specific to the combined activation or silence of multiple triggers. For instance, followers can emerge from the simultaneous activation of two triggers but are absent otherwise. More generally, the propagation of sequential activation is sensitive to contextual activity in two principal ways. In the majority of cases, concurrent sequences inhibit each other. In some cases, however, contextual neurons can selectively and reliably direct activity along alternative paths. 

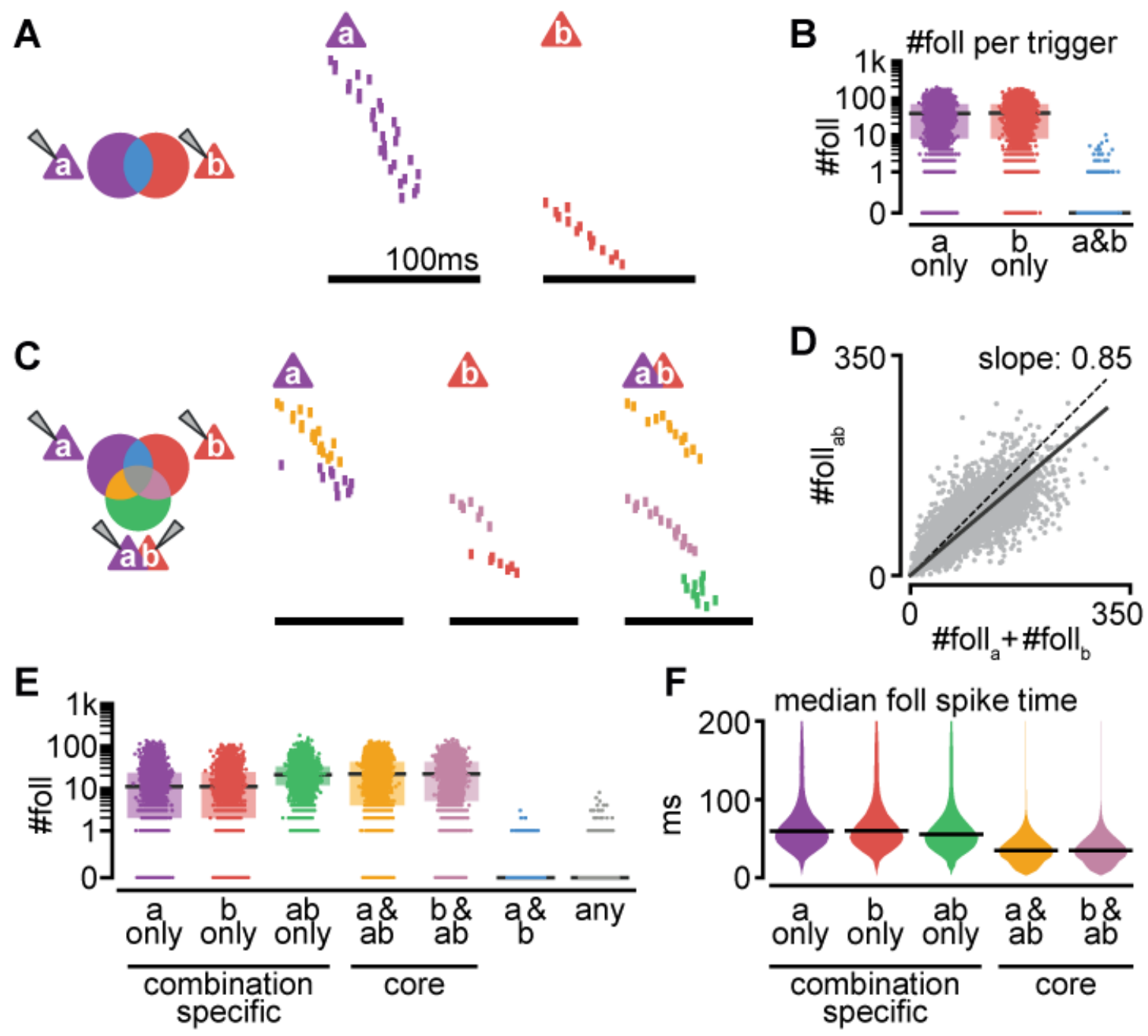

G
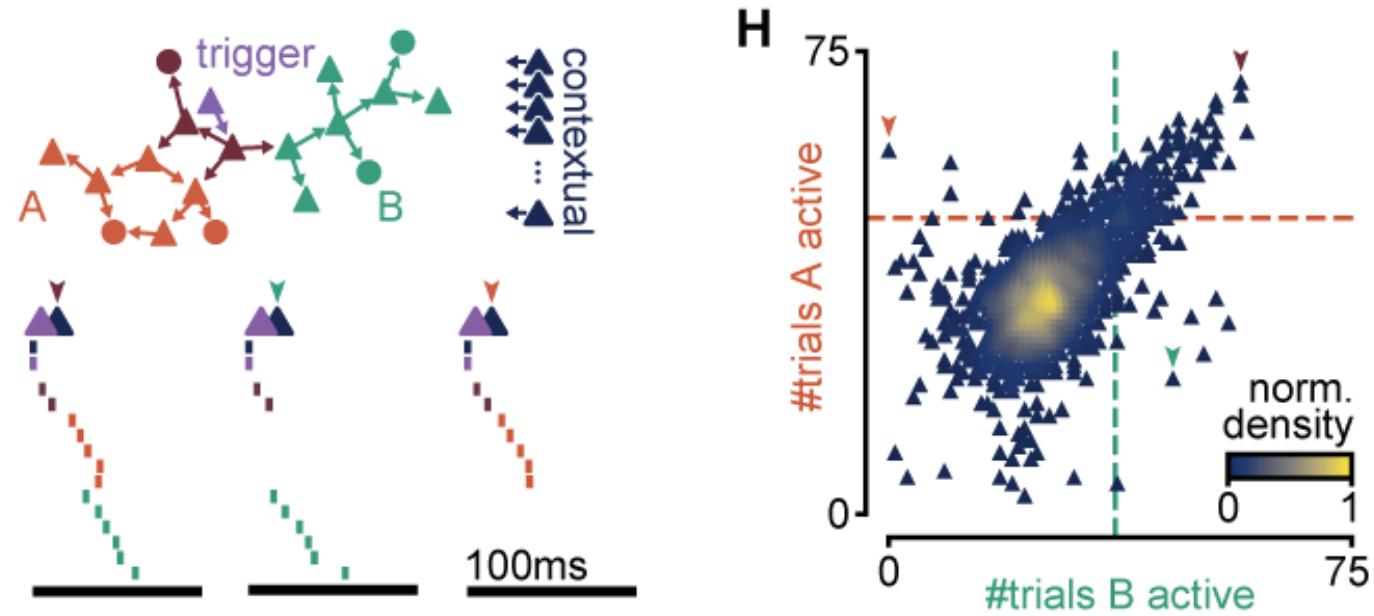

Fig. 6: Interactions between sequences triggered by different neurons.

A. Left: schematic of follower classes as a function of trigger neuron (purple: follower to a; red: follower to b; blue: follower to both $a$ and $b$ ). Right: example sequences produced by the activation of $a$ or $b$. Followers are sorted by trigger class (left) and median spike time. Triggers do not share any followers (blue trigger class).

B. Number of followers for each trigger class in $\mathbf{A}$ for all random pairs of simulations 
$(n=5,000)$. Boxes: median and $\left[25^{\text {th }}, 75^{\text {th }}\right]$ percentiles.

C. Left: schematic of follower classes as a function of trigger: single model neuron ( $a$ or $b$ ) or simultaneous coactivation (a\&b). Right: same trials as in $\mathbf{A}$, and a trial under coactivation. Followers are sorted by trigger class (left) and median spike time. Same follower sorting in all trials.

D. Number of followers present under trigger coactivation as a function of the sum of followers for single triggers. Dashed line: diagonal. Solid line: linear fit with zero intercept.

E. Number of followers for each trigger class in $\mathbf{C}$ for all random pairs of triggers $(n=5,000)$.

F. Median follower spike time for each trigger class in $\mathbf{C}$ after pooling followers from all simulations (5,000 simulations; 532,208 followers). Lines indicate median.

G. Top: Trigger neuron (purple), followers, strong connections between them (arrows), and other neurons in the network (dark blue). Follower colors as in Fig. 4B. Bottom: sequences triggered under simultaneous coactivation of trigger (purple) and one additional contextual neuron that facilitates propagation in both sub-networks (left), only in the green subnetwork (middle) or only in the orange sub-network (right).

$\mathbf{H}$. Effect on green and orange sub-network activation ( $\mathbf{G}$ top) when the trigger neuron is coactivated with each of 2,000 randomly selected contextual neurons. Dashed lines indicate baseline activation of each sub-network. Neurons are colored by local density. Colored arrows correspond to sequences in $\mathbf{G}$ bottom.

\section{Discussion}

Inspired and guided by experimental data in the turtle cortex, we developed a model to investigate how activity triggered by a single spike can propagate reliably in a recurrent network. Our model produces sequences as experimentally measured ex vivo in the turtle cortex and predicts their existence in vivo, where firing rates are higher, with marked differences between excitatory and inhibitory followers. We found that strong but rare connections form a substrate for reliable propagation in the model network, while dense but weak connections could enhance or disrupt propagation. Studying propagation failure revealed that sequences could be decomposed into multiple sub-sequences, each reflecting a sub-network of strongly connected followers. We showed that activation of individual subsequences is sensitive to the state of a few gate neurons and can thus be externally controlled. We observed that trigger neurons rarely shared followers but that their sequences often interacted. Followers could be specific to the combination of activation or silence of multiple triggers. Finally, we found that the exact path of propagation is influenced by contextual activity provided by the activation of a small percentage of other neurons in the network.

\section{Routing}

The sequences in our experiments and model represent an example of propagating cortical activity. They are related to, but distinct from, previous frameworks: temporary breaking of excitatory/inhibitory balance, synfire chains, neuronal avalanches, and stochastic resonance.

Our work suggests that the disruption of propagating spiking sequences may be a landmark of flexibility, where apparent failures to propagate activity are the consequence of routing it (Fig. 7). Consequently, followers with low firing rate modulation could be alternative recipients of activity. The path of propagation and the recipient of activity might differ across 
trials due to changes in contextual activity. We propose that the dynamic routing of activity relies on two elements: the existence, for each path, of few points of failure and specific mechanisms to manipulate them. The strength of some connections in our model enables reliable propagation, while their sparsity defines groups of neurons with few entry connections (gates). Precisely-timed external inputs can selectively open or close these gates. In addition, ongoing parallel sequences can also influence the path of propagation in the form of lateral inhibition (competition) or excitation (cooperation) between sub-networks in the recurrent network. We expect that complex computations can be built from our proposed small-scale form of gating. Previous theoretical work on signal propagation in recurrent networks with irregular background activity found that the combination of parallel pathways and gating can implement different logic statements, illustrating how gating can form the basis for more complex computations (Vogels and Abbott, 2005).

Our experiments with the simultaneous activation of multiple triggers suggested that sequences operate under excitatory/inhibitory balance, where excitatory signals are canceled by local inhibition (Hemberger et al., 2019). We did not explicitly construct our model to be balanced. Still, this form of lateral inhibition and routing via the alteration of excitatory and inhibitory conductances in gate neurons are reminiscent of "precise balance", a form of balance within short temporal windows (observed in hippocampal slices (Bhatia et al., 2019)). Hence, our work is consistent with and extends previous theoretical work proposing that the gating of firing rate signals could be arbitrated via the disruption of excitatory/inhibitory balance in local populations (Vogels and Abbott, 2009).

Synfire chains are a classical model of reliable propagation (Abeles, 1991; Diesmann et al., 1999). While the biological evidence for synfire chains is inconclusive (Abeles et al., 1993; Egger et al., 2020; Fiete et al., 2010; Oram et al., 1999; Prut et al., 1998), theoretical studies have embedded them in recurrent networks yielding insights into the properties of stimuli best suited for propagation (Kremkow et al., 2010; Kumar et al., 2010). Contrary to classical synfire chains, activity within our networks propagates over thin synaptic chains (sub-networks), which are powerful enough to make the synchronous firing of large groups of neurons unnecessary. Furthermore, $80-95 \%$ of excitatory neurons in our experiments and model could trigger sequences, suggesting that thin chains of strong connections are common within turtle cortex. Consequently, reliable propagation of activity may involve much fewer neurons and spikes than initially predicted by synfire models.

In our experiments and model, a single spike could trigger multiple ones, but without runaway excitation, suggesting that sequences share some properties with neuronal avalanches (Beggs and Plenz, 2003). Criticality, a dynamical regime that can maximize information transmission in recurrent networks (Beggs, 2008), has been associated with the ex vivo turtle cortex after observing scale-free statistics of population activity (Shew et al., 2015). Although cortical criticality does not require reliability, it does not preclude it. Indeed, repeatable and precise LFP avalanches have been found in slice cultures, possibly reflecting the existence of underlying repeatable spiking sequences (Beggs and Plenz, 2004). Our finding of spike sequences as the expression of routing by strong connections may thus be an alternative view, compatible with the hypothesis of criticality in cortical circuits. Importantly, we interpret the halting of sequences as a form of contextual gating. While avalanches may statistically describe sequence sizes as a stochastic branching process, our simulations with fixed external input (Fig. 5) and contextual activity (Fig. 6) suggest that reliable control of sub-sequences is possible. Consequently, network states that remain constant from trial to trial will produce sequence statistics that differ from pure branching- 
bioRxiv preprint doi: https://doi org/10.1101/2021.1221.473652; this version posted December 23, 2021. The copyright holder for this preprint (which was not certified by peer review) is the author/funder, who has granted bioRxiv a license to display the preprint in perpetuity. It is made available under aCC-BY-NC-ND 4.0 International license.

process statistics (e.g., powerlaws). Experimentally, this might be achieved pharmacologically or by increasing the number of neurons that are coactivated with sequence triggers as in Fig. 6G-H.

Lastly, stochastic resonance is a phenomenon where noise can enhance the response of a system (Collins et al., 1995a). In particular, long-tailed distributions of strengths may create the opportunity for aperiodic stochastic resonance between weak and strong spike inputs (Collins et al., 1995b; Teramae et al., 2012). Indeed, our simulations with and without weak connections revealed narrow ranges of input where they could increase or decrease reliability (Fig. $\mathbf{3 H}$ ). This global mechanism of control contrasts with the selectivity of single sub-networks to the activation of a few neurons within the recurrent circuit (Fig. $6 \mathbf{H}$ ). As with criticality and synfire chains, our results suggest that some routing mechanisms might not be stochastic and operate at the population-level, but rather that they are reliable and finegrained. Importantly, our model exhibited these features even under random connectivity and spontaneous drive.

\section{Connectivity}

Our experimental and modeling results show that rare but strong connections are key to the generation of sequential activity in the cortex of turtles. Such sparse and strong connections are a common feature of brain connectivity. Indeed, long-tailed distributions of connection strengths are found in rat visual cortex (Song et al., 2005), mouse barrel and visual cortices (Cossell et al., 2015; Lefort et al., 2009), rat and guinea pig hippocampus (Ikegaya et al., 2013; Sayer et al., 1990) and human cortex (Shapson-Coe et al., 2021). Modeling and in vitro studies in rat hippocampus and piriform cortex have suggested that sparse strong inputs substantially affect their postsynaptic partners and network dynamics (Franks and Isaacson, 2006; Ikegaya et al., 2013). Furthermore, repeatable sequences of action potentials have also been reported in most of these mammalian cortical structures (Buzsáki and Tingley, 2018; Carrillo-Reid et al., 2015; Dechery and MacLean, 2017; Diba and Buzsáki, 2007; Dragoi and Tonegawa, 2011; Luczak and MacLean, 2012; Luczak et al., 2015; Vaz et al., 2020). Thus, could strong connections underlie cortical sequences more generally, and are sequences an ancient feature of cortical computation?

Our study identified distinct roles for strong and weak connections, providing reliability and flexibility, respectively. Experimental distributions of trial-to-trial variability of EPSP sizes in rat and mouse cortices show that the amplitude of large EPSPs is less variable than that of small EPSPs, supporting the greater reliability of strong connections (Buzsáki and Mizuseki, 2014; Ikegaya et al., 2013; Lefort et al., 2009). Recent electron microscopy evidence from mouse primary visual cortex (V1) shows that the distribution of synapse sizes between L2/3 pyramidal neurons is best described by the combination of two lognormal distributions (Dorkenwald et al., 2019). This binarization of excitatory synapse types may be the morphological equivalent of our modeling predictions.

Although we based many model parameters on experimental data (connection strengths, single-neuron properties, connection probabilities), our model was otherwise random. Consequently, the model displays a high degree of heterogeneity, allowing us to make nontrivial and general predictions. However, other features of cortical organization, such as structured connectivity, neuronal diversity, and dendritic interactions, might affect activity propagation and routing. Indeed, certain nonrandom features may be expected in the connectivity of the turtle visual cortex, such as axonal projection biases (Shein-Idelson et al., 
2017), differences between apical and basal dendritic connectivity (Fournier et al., 2015), or plasticity-derived structures. Transcriptomic and morphological evidence suggests that turtle cortex contains diverse neuronal types, as seen in mammalian cortex (Nenadic et al., 2003; Tosches et al., 2018). Different neuronal types may play different roles in information routing. For instance, somatostatin- and parvalbumin-expressing neurons in mouse $\mathrm{V} 1$ may play different roles in controlling trial-to-trial reliability (Rikhye et al., 2021). Finally, our model represents neurons as single compartments, but complex input interactions and nonlinearities may occur at the level of dendritic arbors. For instance, synapses may cluster in short dendritic segments to effectively drive postsynaptic spiking (Kirchner and Gjorgjieva, 2021; Scholl et al., 2021); these inputs may be locally balanced by inhibition (lascone et al., 2020); and sequential activation within clusters may be particularly effective at triggering somatic spiking (Ishikawa and Ikegaya, 2020). The consequences of these aspects of cortical organization on information routing via strong connections remain to be explored.

\section{Function}

Our model predicts the existence of sequences under in vivo levels of baseline activity. Because our experiments were carried out ex vivo, we lack a direct experimental link between sequences and behavior. Evidence from other animal models has commonly associated spiking sequences to memory or spatial tasks (Buzsáki and Tingley, 2018; Diba and Buzsáki, 2007; Dragoi and Tonegawa, 2011; Modi et al., 2014; Vaz et al., 2020), or reported sequences within the spontaneous or stimulus-evoked activity in sensory areas (Carrillo-Reid et al., 2015; Dechery and MacLean, 2017; Fellous et al., 2004; Luczak and MacLean, 2012; Luczak et al., 2015). The dorsal cortex of turtles is a visual processing area that shows no clear retinotopy (Fournier et al., 2018) but displays stimulus-evoked wave patterns (Nenadic et al., 2003; Prechtl et al., 1997). Most neurons have receptive fields covering the entire visual field and display a wide range of orientation selectivity (Fournier et al., 2018). The presence of orientation tuning in the turtle cortex and our result that excitatory neurons are mainly activated via strong connections (Fig. 3C-D) predict that like-tuned neurons should share strong connections. Interestingly, long-tailed distributions of connection strengths between L2/3 neurons of mouse V1 play a prominent role in orientation tuning in vivo (Cossell et al., 2015) (but see opposite evidence in ferret V1 (Scholl et al., 2021)). There, stimulus presentation triggers broadly-tuned depolarization via a majority of weak connections, while sparse strong connections determine orientation-selective responses (Cossell et al., 2015). This untuned depolarization via weak connections is consistent with our finding of regimes of activity where weak connections can modulate the reliability of propagation (Fig. $\mathbf{3 G}$ ). Overall, the dual roles in routing played by weak and strong connectivity may have corresponding roles in what we describe as tuning. "Tuning" is an operational term, describing a slice of the recurrent pipeline of transformations linking stimulus to perception or behavior. Routing of spikes and tuning thus likely represent different ways of describing the same processes.

Our experiments with multiple-trigger neuron activation revealed non-additive effects on follower composition (Hemberger et al., 2019) that we can mechanistically explain as interactions between sub-networks of strong connections. In our model, the scale of the temporal windows for effective single-sub-network gating was about twice as long as the standard deviation of follower spike times (approximately $100 \mathrm{~ms}$ and $50 \mathrm{~ms}$, respectively, Fig. 5C and Fig. 2H). Consequently, parallel running sequences (e.g., as observed in the mouse auditory cortex (Dechery and MacLean, 2017)) may have the temporal precision to 
interact consistently across trials. Indeed, our model produced late-activated followers that were reliable and specific to the coactivation of two trigger neurons (Fig. 6E). The presence of combination-specific followers in our model suggests that the tuning of a neuron may be expressed as the result of logical operations on the tuning properties of other neurons in the recurrent circuit. Under this view, lateral inhibition between sub-networks in a sensory cortex may perform nontrivial computations on the properties of stimuli. Indeed, lateral inhibition triggered by early responding principal neurons implements concentration-invariant coding in mouse olfactory cortex (Bolding and Franks, 2018). In line with our observation of common lateral inhibition between sub-networks (Fig. 5E, Fig. $6 \mathbf{H}$ ), activations of single neurons in mouse V1 tend to inhibit neurons with overlapping tuning (feature competition) (Chettih and Harvey, 2019). Similarly, highly specific lateral excitation between sub-networks (Fig. 6H) may act as a mechanism to bind together stimulus properties (Abeles et al., 2004). Given the density of excitatory neurons and the frequent presence of strong connections (Fig. 1A, Fig. 1E), such circuits may display a high combinatorial capacity (Bienenstock, 1995).

In conclusion, our results establish a mechanistic link between three elements common to most cortical circuits: sparse firing, spiking sequences, and long-tailed distributions of connection strengths. We provide a computational interpretation for spiking sequences as the expression of reliable but flexible routing of single spikes over sets of neurons connected by strong connections (Fig. 7). By dissecting how a cortex responds to single spikes, we illustrate the relevance of this elementary unit of communication between neurons in cortical computations.
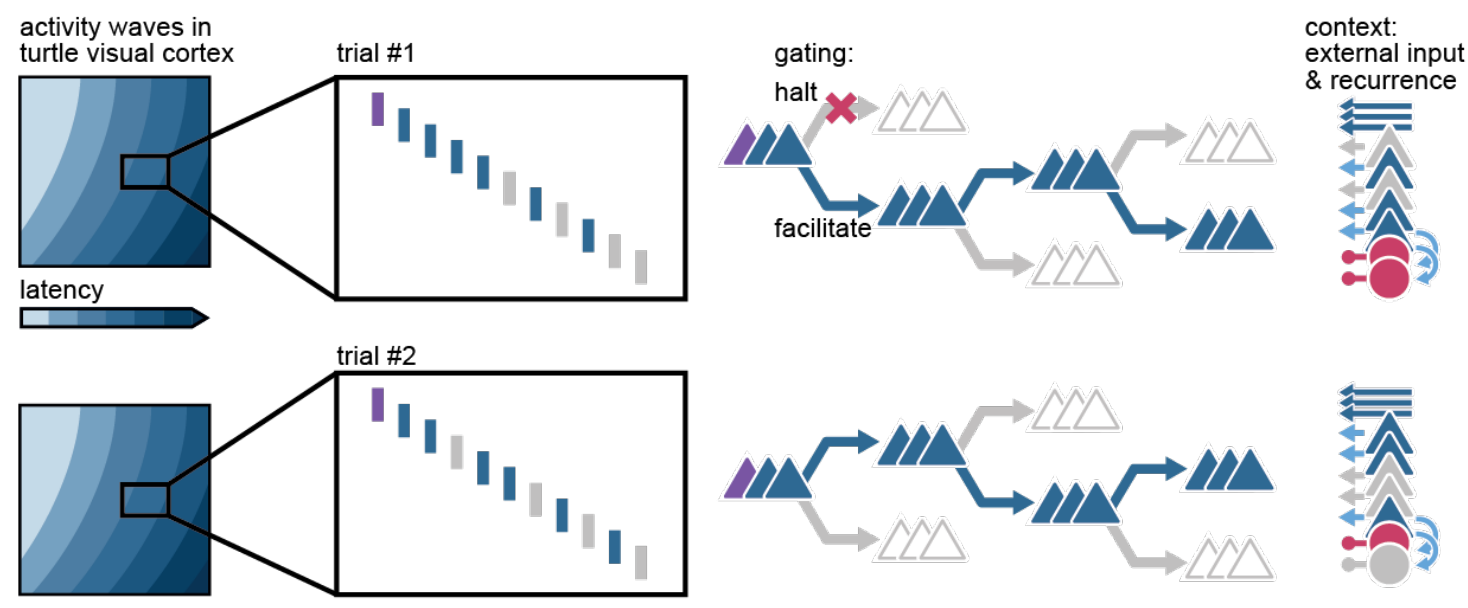

Fig. 7. Routing using sparse strong connectivity.

As activity propagates in the turtle visual cortex, a spike will preferentially propagate through strong connections. The strength of those connections contributes to the reliability of propagation, while their sparsity creates gates where propagation can be halted or facilitated, introducing flexibility. The halting of activity will appear as partial failures of the expected sequence of spikes over different initial neuron activations. The spiking state of other neurons in the recurrent network, together with external inputs, form a dynamical context that determines the final path for each spike.

\section{Methods}




\section{Neuron and synapse models}

We simulated neurons as adaptive exponential integrate-and-fire (see Table 1 for parameters) (Brette and Gerstner, 2005). Membrane leak conductance and capacitance were fitted using least-squares from current-clamp traces obtained in whole-cell clamp under different levels of current injection (Hemberger et al., 2019). We used the median of the distributions for all neurons in the model. Membrane parameters resulted in a rheobase current of $150 \mathrm{pA}$ for a model neuron in isolation.

We fitted synaptic time constants to rise times of excitatory postsynaptic potentials (EPSPs) obtained in paired patch recordings (same as Fig. 1D) (Hemberger et al., 2019) using least-squares and used the median for our model synapses. We converted experimentally measured EPSP amplitudes to synaptic conductances (Fig. 1D) using least squares and fitted a lognormal distribution via maximum likelihood estimation. When drawing strengths from the resulting lognormal distribution, we discarded and re-sampled conductances higher than the maximum conductance of all fitted EPSP amplitudes (67.8 nS, $21 \mathrm{mV}$ ). Inhibitory synaptic conductances were drawn from the same truncated lognormal distribution and scaled up by 8 , resulting in currents 2.5 times stronger and a maximum IPSP amplitude of $-21 \mathrm{mV}$.

\section{Table 1: Neuron and synapse model parameters.}

Asterisk $\left({ }^{*}\right)$ indicates parameters fitted from experimental data (Hemberger et al., 2019). Parameters of excitatory synaptic conductance are for a truncated lognormal distribution.

\begin{tabular}{|l|l|}
\hline Variable & Value \\
\hline Excitatory reversal potential & $10 \mathrm{mV}$ \\
\hline Inhibitory reversal potential & $-75 \mathrm{mV}$ \\
\hline Synaptic conductance time constant ${ }^{*}$ & $1.103681 \mathrm{~ms}$ \\
\hline Excitatory synaptic conductance $(\mathrm{mean})^{*}$ & $3.73 \mathrm{nS}$ \\
\hline Excitatory synaptic conductance $(\text { std })^{*}$ & $6.51 \mathrm{nS}$ \\
\hline Excitatory Synaptic conductance $(\mathrm{max})^{*}$ & $67.8 \mathrm{nS}$ \\
\hline Leak reversal potential & $-70.6 \mathrm{mV}$ \\
\hline Spike detection threshold & $0 \mathrm{mV}$ \\
\hline Membrane reset potential & $-60 \mathrm{mV}$ \\
\hline Spike initiation threshold & $-50.4 \mathrm{mV}$ \\
\hline Membrane capacitance* & $239.8 \mathrm{pF}$ \\
\hline Leak conductance* & $4.2 \mathrm{nS}$ \\
\hline Subthreshold adaptation & $4 \mathrm{nS}$ \\
\hline
\end{tabular}




\begin{tabular}{|l|l|}
\hline Spike-triggered adaptation & $80.5 \mathrm{pA}$ \\
\hline Adaptation time constant & $144 \mathrm{~ms}$ \\
\hline Slope factor & $2 \mathrm{mV}$ \\
\hline Refractory period & $2 \mathrm{~ms}$ \\
\hline
\end{tabular}

\section{Network}

Our network model consisted of 93,000 excitatory neurons and 7,000 inhibitory neurons that were randomly assigned a location on a square of side $2,000 \mu \mathrm{m}$.

Neurons were connected randomly. The probability of connection between two given neurons changed as a function of Euclidean distance in the plane, assuming periodic boundary conditions. We first estimated connection probability at regular distance intervals from 918 paired whole-cell patch-clamp recordings (Fig. 1C)(Hemberger et al., 2019). We then fitted these estimates with a Gaussian profile using least squares. Finally, we scaled the profile so that the expected probability within a $200 \mu \mathrm{m}$ radius matched populationspecific probabilities measured experimentally (Fig. 1A). Note that inhomogeneities in neuron location and randomness of connectivity meant that neurons received a variable number of connections.

All strengths and delays were assigned independently for each connection. Neurons did not have autapses.

\section{Simulations}

Simulations were run using Nest 2.16 (Linssen et al., 2018) with a time step of $0.1 \mathrm{~ms}$. Each simulation took between 30-45 minutes on an Intel(R) Xeon(R) Gold 6152 CPU @ 2.10GHz.

We instantiated 300 networks and generated 20 simulations of each instantiation, yielding a batch of 6,000 simulations. Each neuron in each simulation received a random current sampled independently every $1 \mathrm{~ms}$ from a Gaussian distribution $\mathrm{N}\left(\mu_{\mathrm{in}}, \sigma_{\mathrm{in}}\right)$. Each simulation had randomly assigned input parameters $\left(\mu_{\text {in }}\right.$ and $\sigma_{\text {in }}$ ). Additionally, a single trigger neuron was randomly chosen in each network and driven to spike at regular intervals (see Methods, Definition of followers). Whenever we re-ran a simulation, we used the same input statistics and trigger neuron unless otherwise stated.

Certain mean input levels $\left(\mu_{\text {in }}\right)$ could lead to self-sustaining activity (Fig. $\left.1 \mathbf{H}\right)$ but only after a few spikes had occurred in the network, possibly resulting in different activity early and late in a simulation. Consequently, we kick-started all our simulations with a volley of spikes sent to a random selection of 500 excitatory neurons in the first $100 \mathrm{~ms}$ and then discarded the initial $1,000 \mathrm{~ms}$.

We analyzed all 6,000 original simulations to identify sequences of spikes (Fig. 2) and sub-sequences (Fig. 4). A random selection of 900 simulations was analyzed to extract connectivity motifs (Fig. 3A-D). A random selection of 2,000 simulations was re-run with truncated connectivity (Fig. 3E-G). To study the effect of external spikes on sub-sequences (Fig. 5A-B), we re-ran a random selection of 2,000 simulations where the gate of one randomly chosen sub-network received an additional external spike. To study how external 
bioRxiv preprint doi: https://doi org/10.1101/2021.1221.473652; this version posted December 23, 2021. The copyright holder for this preprint (which was not certified by peer review) is the author/funder, who has granted bioRxiv a license to display the preprint in perpetuity. It is made available under aCC-BY-NC-ND 4.0 International license.

spike parameters affected sub-sequences (Fig. 5C-E, Supplementary Fig. 2), we selected 8 simulations and re-ran each one between 1,142 and 3,655 times while varying external spike strength and timing. To study the effect of coactivation of multiple trigger neurons (Fig. 6), we selected a representative simulation (same as Fig. 4A) and re-ran it 7,000 times with new randomly chosen triggers (but same input parameters, $\mu_{\text {in }}$ and $\sigma_{\text {in }}$ ). We repeated this analysis on another 50 randomly chosen simulations re-ran 800 times each

(Supplementary Fig. 3).

\section{Definition of followers}

Every 400 milliseconds, for 100 trials, we set the voltage of the trigger model neuron above the spike detection threshold, generating an instantaneous spike (trials). For each trial, we defined a window before spike injection $(100 \mathrm{~ms})$ and a window after spike injection (300 ms). We computed the mean firing rate for all windows before and after spike injection for all other neurons in the network and defined the difference of firing rate before and after as the firing rate modulation of each neuron $(\Delta \mathrm{FR})$. We normalized $\Delta \mathrm{FR}$ by dividing it by that expected from a neuron that never spiked before spike injection and that spiked precisely once after spike injection.

We defined a null distribution for firing rate modulation $(\Delta F R)$ as the difference of two random samples from two Poisson random variables with the same underlying rate. The rates of the two Poisson random variables were adjusted to account for the different duration of the time windows before and after spike injection. The underlying rate was taken as the mean firing rate of all model neurons in the windows before spike injection. The resulting null distribution was centered at zero but displayed a non-zero variance. We used $p<10^{-7}$ as an upper boundary to the null distribution to segregate spurious firing rate increases from reliable neurons. Since we tested all $10^{5}$ neurons in our model (except the trigger neuron), we expected one false positive on average every 100 simulations. Due to big differences in firing rates, we computed different null distributions for the excitatory and inhibitory populations.

\section{Lognormal fit bootstrap}

We generated 50,000 bootstrapped samples of the probability that a neuron had at least one strong excitatory-to-excitatory connection (Fig. 1F). A connection was strong if it fell between 50.6 and $67.8 \mathrm{nS}$, corresponding, respectively, to the top $99.7 \%$ of the original fitted lognormal distribution (Fig. 1D) and the maximum possible strength.

The number of strong connections $s_{i j}$ of a model neuron $i$ in bootstrap step $j$ follows a Binomial distribution $B\left(n_{i}, p_{j}\right)$, where $n_{i}$ is the total number of connections of neuron $i$, and $p_{j}$ is the probability that a connection falls within the strong weight range. To estimate $p_{j}$ we sampled 122 times, with replacement, from our set of 122 experimentally obtained EPSP amplitudes and fitted a new truncated lognormal. Our model neurons received a variable number of connections $n_{i}$ (see Methods, Network), which was well described by a discretized Gaussian distribution $N(\mu=745, \sigma=27)$. For each bootstrapped step $j$, we thus generated 1,000 values of $n_{i}$, drew one sample $s_{i j}$ of each $B\left(n_{i}, p_{j}\right)$, and calculated the ratio of all $s_{i j}$ bigger or equal to 1 .

\section{Entropy of follower rank}

To compare the reliability of the order of follower activation to that seen in experiments, we used the same entropy-based measure as used first in experiments(Hemberger et al., 
2019) (Fig. 2D). Due to our exploration of different firing rate levels, we often observed sequence failure (Fig. 4) and numbers of followers (Fig. 2F) much higher than the number of trials (100), both factors that limited our capacity to estimate order entropy accurately. Consequently, we limited our estimates to simulations with at least as many trials as followers and to trials where at least $25 \%$ of followers were present.

\section{Graph visualization}

All graphs of strong connections (Fig. 4B-D, Fig. 5C, Fig. 5E, Fig. 6G, Supplementary Fig. 2B) were laid out using Graphviz dot and do not represent the spatial location of model neurons unless otherwise specified.

\section{Electrophysiology}

All electrophysiological recordings used to fit the models were described in the original experimental study (Hemberger et al., 2019).

Adaptation index was computed as the ratio of inter-spike-interval between the last two spikes and the interspike interval of the first two spikes in whole-cell patch current-clamp recordings under a constant current injection for one second. The current value was 2-5 times the current value that elicited a single spike for each particular neuron.

\section{References}

Abeles, M. (1991). Corticonics: Neural Circuits of the Cerebral Cortex (Cambridge University Press).

Abeles, M., Bergman, H., Margalit, E., and Vaadia, E. (1993). Spatiotemporal firing patterns in the frontal cortex of behaving monkeys. J. Neurophysiol. 70, 1629-1638.

Abeles, M., Hayon, G., and Lehmann, D. (2004). Modeling compositionality by dynamic binding of synfire chains. J. Comput. Neurosci. 17, 179-201.

Bair, W., and Koch, C. (1996). Temporal precision of spike trains in extrastriate cortex of the behaving macaque monkey. Neural Comput. 8, 1185-1202.

Beggs, J.M. (2008). The criticality hypothesis: how local cortical networks might optimize information processing. Philos. Trans. R. Soc. Math. Phys. Eng. Sci. 366, 329-343.

Beggs, J.M., and Plenz, D. (2003). Neuronal Avalanches in Neocortical Circuits. J. Neurosci. 23, 11167-11177.

Beggs, J.M., and Plenz, D. (2004). Neuronal avalanches are diverse and precise activity patterns that are stable for many hours in cortical slice cultures. J. Neurosci. 24, 5216.

Bhatia, A., Moza, S., and Bhalla, U.S. (2019). Precise excitation-inhibition balance controls gain and timing in the hippocampus. ELife 8 , e43415.

Bienenstock, E. (1995). A model of neocortex. Netw. Comput. Neural Syst. 6, 179-224.

Bolding, K.A., and Franks, K.M. (2017). Complementary codes for odor identity and intensity in olfactory cortex. ELife 6, e22630.

Bolding, K.A., and Franks, K.M. (2018). Recurrent cortical circuits implement concentrationinvariant odor coding. Science 361 , eaat6904. 
bioRxiv preprint doi: https://doi org/10.1101/2021.1221.473652; this version posted December 23, 2021. The copyright holder for this preprint (which was not certified by peer review) is the author/funder, who has granted bioRxiv a license to display the preprint in perpetuity. It is made available under aCC-BY-NC-ND 4.0 International license.

Brecht, M., Schneider, M., Sakmann, B., and Margrie, T.W. (2004). Whisker movements evoked by stimulation of single pyramidal cells in rat motor cortex. Nature 427, 704-710.

Brette, R. (2015). Philosophy of the Spike: Rate-Based vs. Spike-Based Theories of the Brain. Front. Syst. Neurosci. 9.

Brette, R., and Gerstner, W. (2005). Adaptive exponential integrate-and-fire model as an effective description of neuronal activity. J. Neurophysiol. 94, 3637-3642.

Buzsáki, G., and Mizuseki, K. (2014). The log-dynamic brain: how skewed distributions affect network operations. Nat. Rev. Neurosci. 15, 264-278.

Buzsáki, G., and Tingley, D. (2018). Space and Time: The Hippocampus as a Sequence Generator. Trends Cogn. Sci. 22, 853-869.

Carrillo-Reid, L., Miller, J. -e. K., Hamm, J.P., Jackson, J., and Yuste, R. (2015). Endogenous sequential cortical activity evoked by visual stimuli. J. Neurosci. 35, 88138828.

Chettih, S.N., and Harvey, C.D. (2019). Single-neuron perturbations reveal feature-specific competition in V1. Nature 567, 334-340.

Collins, J.J., Chow, C.C., and Imhoff, T.T. (1995a). Stochastic resonance without tuning. Nature 376, 236-238.

Collins, J.J., Chow, C.C., and Imhoff, T.T. (1995b). Aperiodic stochastic resonance in excitable systems. Phys. Rev. E 52, R3321-R3324.

Cossell, L., lacaruso, M.F., Muir, D.R., Houlton, R., Sader, E.N., Ko, H., Hofer, S.B., and Mrsic-Flogel, T.D. (2015). Functional organization of excitatory synaptic strength in primary visual cortex. Nature 518, 399-403.

Dechery, J.B., and MacLean, J.N. (2017). Emergent cortical circuit dynamics contain dense, interwoven ensembles of spike sequences. J. Neurophysiol. 118, 1914-1925.

Diba, K., and Buzsáki, G. (2007). Forward and reverse hippocampal place-cell sequences during ripples. Nat. Neurosci. 10, 1241-1242.

Diesmann, M., Gewaltig, M.-O., and Aertsen, A. (1999). Stable propagation of synchronous spiking in cortical neural networks. Nature 402, 529-533.

Dorkenwald, S., Turner, N.L., Macrina, T., Lee, K., Lu, R., Wu, J., Bodor, A.L., Bleckert, A.A., Brittain, D., Kemnitz, N., et al. (2019). Binary and analog variation of synapses between cortical pyramidal neurons (Neuroscience).

Doron, G., von Heimendahl, M., Schlattmann, P., Houweling, A.R., and Brecht, M. (2014). Spiking irregularity and frequency modulate the behavioral report of single-neuron stimulation. Neuron 81, 653-663.

Dragoi, G., and Tonegawa, S. (2011). Preplay of future place cell sequences by hippocampal cellular assemblies. Nature 469, 397-401.

Egger, R., Tupikov, Y., Elmaleh, M., Katlowitz, K.A., Benezra, S.E., Picardo, M.A., Moll, F., Kornfeld, J., Jin, D.Z., and Long, M.A. (2020). Local Axonal Conduction Shapes the Spatiotemporal Properties of Neural Sequences. Cell 183, 537-548.e12. 
bioRxiv preprint doi: https:/doi org/10.1101/2021.1221.473652; this version posted December 23,2021 . The copyright holder for this preprint (which was not certified by peer review) is the author/funder, who has granted bioRxiv a license to display the preprint in perpetuity. It is made available under aCC-BY-NC-ND 4.0 International license.

Fellous, J.-M. (2004). Discovering Spike Patterns in Neuronal Responses. J. Neurosci. 24, 2989-3001.

Fellous, J.-M., Tiesinga, P.H., Thomas, P.J., and Sejnowski, T.J. (2004). Discovering spike patterns in neuronal responses. J. Neurosci. 24, 2989-3001.

Fiete, I.R., Senn, W., Wang, C.Z.H., and Hahnloser, R.H.R. (2010). Spike-time-dependent plasticity and heterosynaptic competition organize networks to produce long scale-free sequences of neural activity. Neuron $65,563-576$.

Fournier, J., Müller, C.M., and Laurent, G. (2015). Looking for the roots of cortical sensory computation in three-layered cortices. Curr. Opin. Neurobiol. 31, 119-126.

Fournier, J., Müller, C.M., Schneider, I., and Laurent, G. (2018). Spatial Information in a Non-retinotopic Visual Cortex. Neuron 97, 164-180.e7.

Franks, K.M., and Isaacson, J.S. (2006). Strong Single-Fiber Sensory Inputs to Olfactory Cortex: Implications for Olfactory Coding. Neuron 49, 357-363.

Hahnloser, R.H.R., Kozhevnikov, A.A., and Fee, M.S. (2002). An ultra-sparse code underlies the generation of neural sequences in a songbird. Nature 419, 65-70.

Hemberger, M., Shein-Idelson, M., Pammer, L., and Laurent, G. (2019). Reliable Sequential Activation of Neural Assemblies by Single Pyramidal Cells in a Three-Layered Cortex. Neuron 104, 353-369.e5.

Houweling, A.R., and Brecht, M. (2008). Behavioural report of single neuron stimulation in somatosensory cortex. Nature 451, 65-68.

lascone, D.M., Li, Y., Sümbül, U., Doron, M., Chen, H., Andreu, V., Goudy, F., Blockus, H., Abbott, L.F., Segev, I., et al. (2020). Whole-Neuron Synaptic Mapping Reveals Spatially Precise Excitatory/Inhibitory Balance Limiting Dendritic and Somatic Spiking. Neuron 106, 566-578.e8.

Ikegaya, Y., Aaron, G., Cossart, R., Aronov, D., Lampl, I., Ferster, D., and Yuste, R. (2004). Synfire chains and cortical songs: temporal modules of cortical activity. Science 304, 559564.

Ikegaya, Y., Sasaki, T., Ishikawa, D., Honma, N., Tao, K., Takahashi, N., Minamisawa, G., Ujita, S., and Matsuki, N. (2013). Interpyramid spike transmission stabilizes the sparseness of recurrent network activity. Cereb. Cortex 23, 293-304.

Ishikawa, T., and Ikegaya, Y. (2020). Locally sequential synaptic reactivation during hippocampal ripples. Sci. Adv. 6, eaay1492.

Izhikevich, E.M. (2006). Polychronization: computation with spikes. Neural Comput. 18, 245282.

Kajiwara, M., Nomura, R., Goetze, F., Akutsu, T., and Shimono, M. (2020). Inhibitory neurons are a Central Controlling regulator in the effective cortical microconnectome (Neuroscience).

Kirchner, J.H., and Gjorgjieva, J. (2021). Emergence of local and global synaptic organization on cortical dendrites. Nat. Commun. 12, 4005. 
Kremkow, J., Aertsen, A., and Kumar, A. (2010). Gating of signal propagation in spiking neural networks by balanced and correlated excitation and inhibition. J. Neurosci. 30, 15760-15768.

Kumar, A., Rotter, S., and Aertsen, A. (2010). Spiking activity propagation in neuronal networks: reconciling different perspectives on neural coding. Nat. Rev. Neurosci. 11, 615627.

Kwan, A.C., and Dan, Y. (2012). Dissection of cortical microcircuits by single-neuron stimulation in vivo. Curr. Biol. 22, 1459-1467.

Lefort, S., Tomm, C., Floyd Sarria, J.-C., and Petersen, C.C.H. (2009). The excitatory neuronal network of the $\mathrm{C} 2$ barrel column in mouse primary somatosensory cortex. Neuron 61, 301-316.

Linssen, C., Lepperød, M.E., Mitchell, J., Pronold, J., Eppler, J.M., Keup, C., Peyser, A., Kunkel, S., Weidel, P., Nodem, Y., et al. (2018). NEST 2.16.0 (Zenodo).

London, M., Roth, A., Beeren, L., Häusser, M., and Latham, P.E. (2010). Sensitivity to perturbations in vivo implies high noise and suggests rate coding in cortex. Nature 466, 123127.

Long, M.A., Jin, D.Z., and Fee, M.S. (2010). Support for a synaptic chain model of neuronal sequence generation. Nature 468, 394-399.

Luczak, A., and MacLean, J.N. (2012). Default activity patterns at the neocortical microcircuit level. Front. Integr. Neurosci. 6.

Luczak, A., McNaughton, B.L., and Harris, K.D. (2015). Packet-based communication in the cortex. Nat. Rev. Neurosci. 16, 745-755.

Maes, A., Barahona, M., and Clopath, C. (2020). Learning spatiotemporal signals using a recurrent spiking network that discretizes time. PLOS Comput. Biol. 16, e1007606.

Modi, M.N., Dhawale, A.K., and Bhalla, U.S. (2014). CA1 cell activity sequences emerge after reorganization of network correlation structure during associative learning. ELife 3, e01982.

Molnár, G., Oláh, S., Komlósi, G., Füle, M., Szabadics, J., Varga, C., Barzó, P., and Tamás, G. (2008). Complex events initiated by individual spikes in the human cerebral cortex. PLoS Biol. 6, e222.

Nenadic, Z., Ghosh, B.K., and Ulinski, P. (2003). Propagating waves in visual cortex: a large-scale model of turtle visual cortex. J. Comput. Neurosci. 14, 161-184.

Oram, M.W., Wiener, M.C., Lestienne, R., and Richmond, B.J. (1999). Stochastic nature of precisely timed spike patterns in visual system neuronal responses. J. Neurophysiol. 81, 3021-3033.

Prechtl, J.C., Cohen, L.B., Pesaran, B., Mitra, P.P., and Kleinfeld, D. (1997). Visual stimuli induce waves of electrical activity in turtle cortex. Proc. Natl. Acad. Sci. 94, 7621-7626.

Prut, Y., Vaadia, E., Bergman, H., Haalman, I., Slovin, H., and Abeles, M. (1998). Spatiotemporal structure of cortical activity: properties and behavioral relevance. J. Neurophysiol. 79, 2857-2874. 
bioRxiv preprint doi: https://doi org/10.1101/2021.1221.473652; this version posted December 23, 2021. The copyright holder for this preprint (which was not certified by peer review) is the author/funder, who has granted bioRxiv a license to display the preprint in perpetuity. It is made available under aCC-BY-NC-ND 4.0 International license.

Rajan, K., Harvey, C.D., and Tank, D.W. (2016). Recurrent Network Models of Sequence Generation and Memory. Neuron 90, 128-142.

Renart, A., de la Rocha, J., Bartho, P., Hollender, L., Parga, N., Reyes, A., and Harris, K.D. (2010). The asynchronous state in cortical circuits. Science 327, 587-590.

Rikhye, R.V., Yildirim, M., Hu, M., Breton-Provencher, V., and Sur, M. (2021). Reliable Sensory Processing in Mouse Visual Cortex through Cooperative Interactions between Somatostatin and Parvalbumin Interneurons. J. Neurosci. 41, 8761-8778.

Rubinski, A., and Ziv, N.E. (2015). Remodeling and Tenacity of Inhibitory Synapses: Relationships with Network Activity and Neighboring Excitatory Synapses. PLOS Comput. Biol. 11, e1004632.

Sayer, R., Friedlander, M., and Redman, S. (1990). The time course and amplitude of EPSPs evoked at synapses between pairs of CA3/CA1 neurons in the hippocampal slice. J. Neurosci. 10, 826-836.

Scholl, B., Thomas, C.I., Ryan, M.A., Kamasawa, N., and Fitzpatrick, D. (2021). Cortical response selectivity derives from strength in numbers of synapses. Nature 590, 111-114.

Shadlen, M.N., and Newsome, W.T. (1994). Noise, neural codes and cortical organization. Curr. Opin. Neurobiol. 4, 569-579.

Shapson-Coe, A., Januszewski, M., Berger, D.R., Pope, A., Wu, Y., Blakely, T., Schalek, R.L., Li, P.H., Wang, S., Maitlin-Shepard, J., et al. (2021). A connectomic study of a petascale fragment of human cerebral cortex (Neuroscience).

Shein-Idelson, M., Pammer, L., Hemberger, M., and Laurent, G. (2017). Large-scale mapping of cortical synaptic projections with extracellular electrode arrays. Nat. Methods 14 882-890.

Shew, W.L., Clawson, W.P., Pobst, J., Karimipanah, Y., Wright, N.C., and Wessel, R. (2015). Adaptation to sensory input tunes visual cortex to criticality. Nat. Phys. 11, 659-663.

Song, S., Sjöström, P.J., Reigl, M., Nelson, S., and Chklovskii, D.B. (2005). Highly nonrandom features of synaptic connectivity in local cortical circuits. PLoS Biol. 3, e68.

Teramae, J., Tsubo, Y., and Fukai, T. (2012). Optimal spike-based communication in excitable networks with strong-sparse and weak-dense links. Sci. Rep. 2, 485.

Tosches, M.A., Yamawaki, T.M., Naumann, R.K., Jacobi, A.A., Tushev, G., and Laurent, G. (2018). Evolution of pallium, hippocampus, and cortical cell types revealed by single-cell transcriptomics in reptiles. Science 360, 881-888.

Vaz, A.P., Wittig, J.H., Inati, S.K., and Zaghloul, K.A. (2020). Replay of cortical spiking sequences during human memory retrieval. Science 367, 1131-1134.

Vogels, T.P., and Abbott, L.F. (2005). Signal propagation and logic gating in networks of integrate-and-fire neurons. J. Neurosci. 25, 10786.

Vogels, T.P., and Abbott, L.F. (2009). Gating multiple signals through detailed balance of excitation and inhibition in spiking networks. Nat. Neurosci. 12, 483-491.

Wehr, M., and Laurent, G. (1996). Odour encoding by temporal sequences of firing in 
bioRxiv preprint doi: https://doi org/10.1101/2021.12 21.473652; this version posted December 23,2021 . The copyright holder for this

preprint (which was not certified by peer review) is the author/funder, who has granted bioRxiv a license to display the preprint in perpetuity. It is made available under aCC-BY-NC-ND 4.0 International license.

oscillating neural assemblies. Nature 384, 162-166.

Wehr, M., and Zador, A.M. (2003). Balanced inhibition underlies tuning and sharpens spike timing in auditory cortex. Nature 426, 442-446.

Wolfe, J., Houweling, A.R., and Brecht, M. (2010). Sparse and powerful cortical spikes. Curr. Opin. Neurobiol. 20, 306-312. 


\section{Supplementary figures}
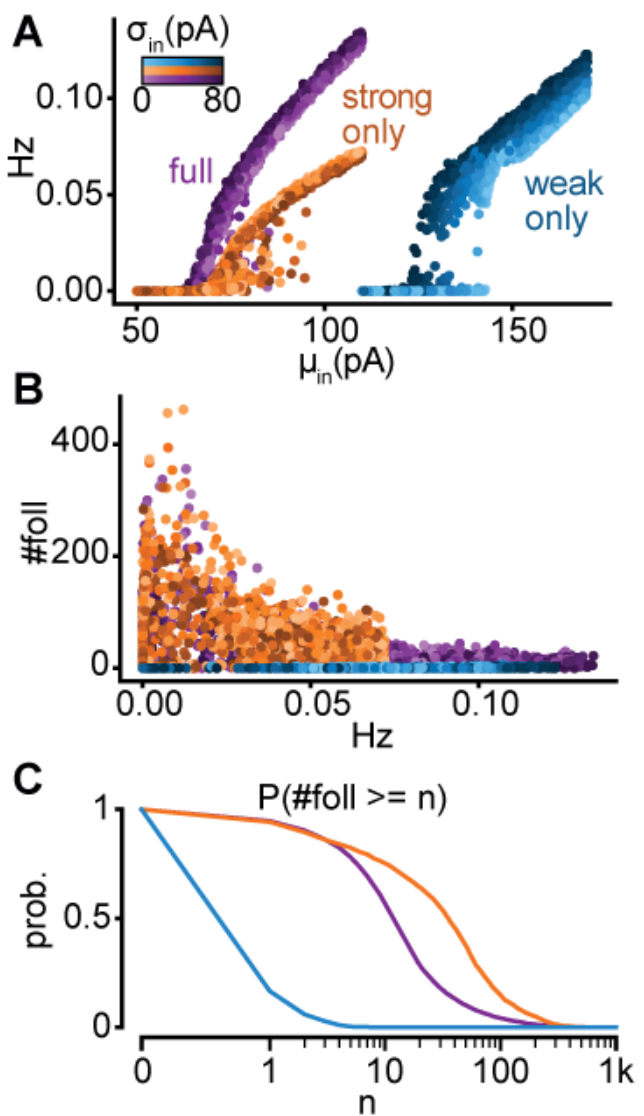

Supplementary Fig. 1: Properties of full, strong-only, and weak-only models.

A. Input-output curves for the full (purple), strong-only (orange), and weak-only (blue) models. Note that the weak-only model produced firing rates only when driven with very strong depolarizations (rheobase current of a model neuron in isolation $\sim 150 \mathrm{pA}$ ).

B. Number of followers as a function of firing rate for each model.

C. Probability of obtaining at least $n$ followers for each model. 
A

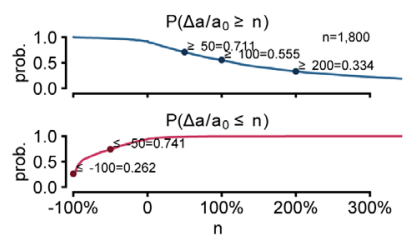

B
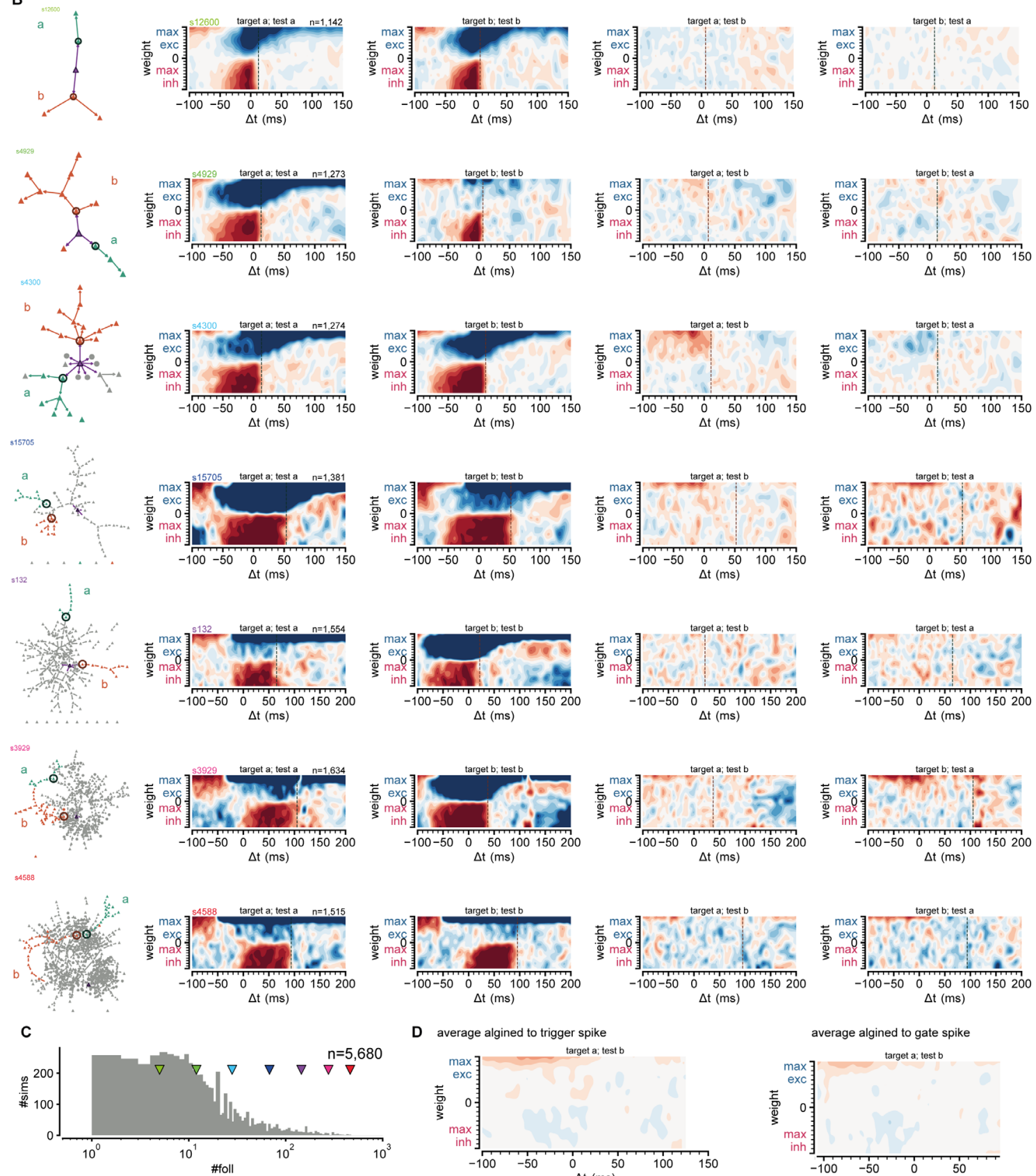

D average algined to trigger spike
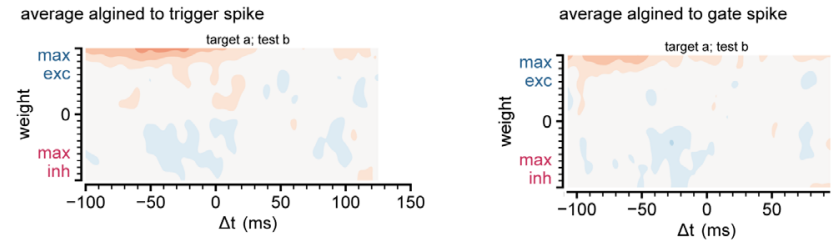

\section{Supplementary Fig. 2: Gating sub-sequence activations}

A. Top: probability of an excitatory input onto a gate neuron producing at least an $\mathrm{n}$-fold increase of activation probability on its respective sub-network. Bottom: same for inhibitory inputs. 
B. Left columns: graph of strong connections between followers for 7 networks with an increasing number of followers. Sub-networks are colored and labeled a and b. Circles indicate sub-network gates. Other columns: change of sub-network activation on subnetwork $a$ or $b$ (test) as a function of external input strength and timing onto the gate of sub-network a or b (target).

C. Number of followers for all networks with at least two sub-networks. Arrowheads indicate networks in $\mathrm{B}$, Baseline firing rates from 0.01 to $0.1 \mathrm{~Hz}$.

D. Left: average change of all maps of sub-network activation in two rightmost columns $\mathbf{B}$. Right: average but aligning all maps to the median spike time of their respective gate.

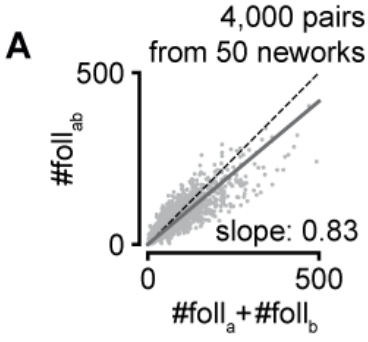

C

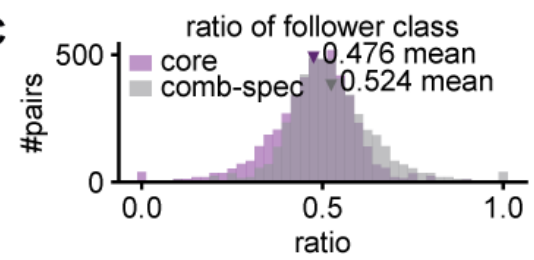

B

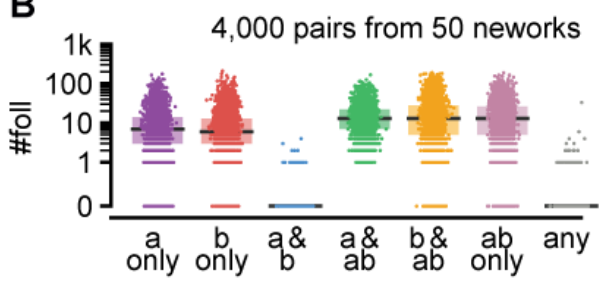

D

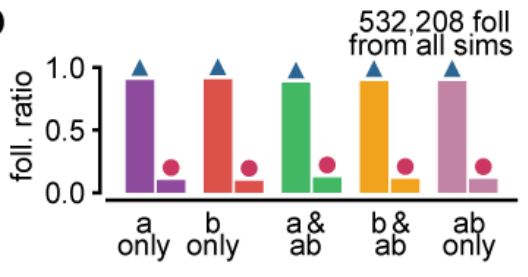

E $2.7 \% 2.9 \% 6.7 \%$ $87.8 \%$

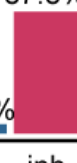

\section{Supplementary Fig. 3: Interactions between sequences.}

A. Number of model followers present under trigger coactivation as a function of the sum of followers for each trigger alone. Same as Fig. 6D but with follower pairs pooled across 50 randomly picked networks (800 pairs per network).

B. Number of followers for each trigger class for all simulations. Same as Fig. 6E but with follower pairs pooled as in $\mathbf{A}$.

C. Ratio of core and combination-specific followers for every pair of random triggers for the network shown in Fig. 6.

D. Ratio of excitatory and inhibitory followers for each trigger class, pooling all followers from the network shown in Fig. 6.

E. Ratio of random triggers for each one of the quadrants in Fig. $6 \mathrm{H}$. Bottom right: $+g-0$; top left: $-\mathrm{g}+0$; top right: exc; bottom left: inh. 